\title{
A [3 + 3] Annelation Approach to (+)-Rhopaloic acid B
}

\author{
Julien C.R. Brioche ${ }^{a}$, Katharine M. Goodenough ${ }^{a}$, David J. Whatrup ${ }^{b}$ and Joseph P.A. Harrity ${ }^{*^{a}}$ \\ a Department of Chemistry, University of Sheffield, Brook Hill, Sheffield, S3 7HF \\ ${ }^{b}$ Synthetic Chemistry, GlaxoSmithKline, Tonbridge, Kent, TN11 9AN, U.K.
}

\section{Supporting Information}

\section{Table of Contents}

Epoxides $\mathbf{6}^{\mathrm{i}}, \mathbf{9}^{\mathrm{ii}}, \mathbf{1 2}^{\mathrm{iii}}$ were prepared according to known procedures.

Preparation/titration of Grignard Reagent 3

Preparation of 7

Preparation of $\mathbf{1 0} \quad 4$

Preparation of $\mathbf{1 3} 5$

Preparation of $\mathbf{1 6} \quad 6$

$\begin{array}{lr}\text { Preparation of } 19 a / b & 6\end{array}$

$\begin{array}{ll}\text { Preparation of } 8 & 7\end{array}$

Preparation of $\mathbf{1 1} 8$

Preparation of $14 \quad 9$

$\begin{array}{ll}\text { Preparation of } \mathbf{1 7} & 10\end{array}$

$\begin{array}{lr}\text { Preparation of 20a } & 10\end{array}$

Preparation of 20b 11

$\begin{array}{ll}\text { Preparation of } \mathbf{2 1} & 12\end{array}$

Preparation of $22 \quad 13$

$\begin{array}{ll}\text { Preparation of } \mathbf{2 3} & 13\end{array}$

$\begin{array}{ll}\text { Preparation of } \mathbf{2 4} & 14\end{array}$

$\begin{array}{lr}\text { Preparation of } \mathbf{2 5} & 15\end{array}$

$\begin{array}{ll}\text { Preparation of } \mathbf{2 6} & 16\end{array}$

$\begin{array}{ll}\text { Preparation of } \mathbf{2 8} & 17\end{array}$

$\begin{array}{lr}\text { Preparation of } 29 & 18\end{array}$

$\begin{array}{lr}\text { Preparation of } \mathbf{3 0} & 19\end{array}$

${ }^{1} \mathrm{H} /{ }^{13} \mathrm{C}$ NMR Spectrum of 7

${ }^{1} \mathrm{H} /{ }^{13} \mathrm{C}$ NMR Spectrum of $\mathbf{1 0} \quad 23$

${ }^{1} \mathrm{H} /{ }^{13} \mathrm{C}$ NMR Spectrum of $\mathbf{1 3} \quad 24$

${ }^{1} \mathrm{H} /{ }^{13} \mathrm{C}$ NMR Spectrum of $\mathbf{1 6} \quad 25$

${ }^{1} \mathrm{H} /{ }^{13} \mathrm{C}$ NMR Spectrum of 19b 26

${ }^{1} \mathrm{H} /{ }^{13} \mathrm{C}$ NMR Spectrum of 19a 27

${ }^{1} \mathrm{H} /{ }^{13} \mathrm{C}$ NMR Spectrum of $8+28$ 
${ }^{1} \mathrm{H} /{ }^{13} \mathrm{C}$ NMR Spectrum of $\mathbf{1 1} \quad 29$

${ }^{1} \mathrm{H} /{ }^{13} \mathrm{C}$ NMR Spectrum of $\mathbf{1 4} \quad 30$

${ }^{1} \mathrm{H} /{ }^{13} \mathrm{C}$ NMR Spectrum of $\mathbf{1 7}$

${ }^{1} \mathrm{H} /{ }^{13} \mathrm{C}$ NMR Spectrum of 20a 32

${ }^{1} \mathrm{H} /{ }^{13} \mathrm{C}$ NMR Spectrum of 20b 33

${ }^{1} \mathrm{H} /{ }^{13} \mathrm{C}$ NMR Spectrum of 22

${ }^{1} \mathrm{H} /{ }^{13} \mathrm{C}$ NMR Spectrum of $\mathbf{2 3} 35$

${ }^{1} \mathrm{H} /{ }^{13} \mathrm{C}$ NMR Spectrum of $\mathbf{2 4} 36$

${ }^{1} \mathrm{H} /{ }^{13} \mathrm{C}$ NMR Spectrum of trans-25

${ }^{1} \mathrm{H} /{ }^{13} \mathrm{C}$ NMR Spectrum of cis-25 38

${ }^{1} \mathrm{H} /{ }^{13} \mathrm{C}$ NMR Spectrum of $\mathbf{2 8} \quad 39$

${ }^{1} \mathrm{H} /{ }^{13} \mathrm{C}$ NMR Spectrum of $29 \quad 40$

Crude ${ }^{1} \mathrm{H}$ NMR Spectrum of intermediate aldehyde $\quad 41$

Crude ${ }^{1} \mathrm{H}$ NMR Spectrum of intermediate enal 42

${ }^{1} \mathrm{H} /{ }^{13} \mathrm{C}$ NMR Spectrum of $\mathbf{3 0} 43$

Comparative data for (+)-rhopaloic acid B 44 


\section{Preparation of the Grignard reagent}

To BuLi (15 mL, $10 \mathrm{M}, 2.1$ eq.) at $0^{\circ} \mathrm{C}$ were added anhydrous ether (114 mL) and TMEDA $(22.6 \mathrm{~mL}, 2.1 \mathrm{eq})$. After cooling the solution at $-78^{\circ} \mathrm{C}$, methallyl alcohol 5 was added dropwise $(6.40 \mathrm{~mL}, 1$ eq. $)$ and the reaction was stirred at $78^{\circ} \mathrm{C}$ for $1 \mathrm{~h}$. The bath was then removed and the resulting mixture was vigorously stirred for $24 \mathrm{~h}$. After this time, stirring was stopped for $30 \mathrm{~min}$ and the solvent was removed via cannula filtration. Anhydrous ether $(100 \mathrm{~mL})$ was then added to the remaining orange solid and the suspension was cooled to $0^{\circ} \mathrm{C}$. At this point a solution of freshly prepared $\mathrm{MgBr}_{2}$ [prepared from magnesium (2.1 eq, $3.9 \mathrm{~g})$, dibromoethane (2.1 eq, $13.8 \mathrm{~mL})$ in ether $(58 \mathrm{~mL})$ ] was transferred quickly via cannula to the suspension. After addition the bath was removed and the suspension was vigorously stirred for 30-45 min. At this stage, the stirring was stopped and the Grignard solution was then separated from colourless solid via cannula. The Grignard solution was titrated and stored in a refrigerator under argon.

\section{Titration of the Grignard reagent ${ }^{\mathrm{iv}}$}

To menthol (31 mg, $0.2 \mathrm{mmol}$ ) in dry THF (1.5 mL) was added 1,10phenanthroline (4 mg). The Grignard reagent to be assayed was added dropwise by syringe until a distinct violet or burgundy colour persisted for longer than one minute.

Concentration $=(\mathrm{mol}$. menthol $\times 1000) /$ vol. Grignard added (usually $0.8-0.15$ M)

\section{Grignard addition to epoxides}

Preparation of 2-methylene-6-phenylhexane-1,5-diol (7).

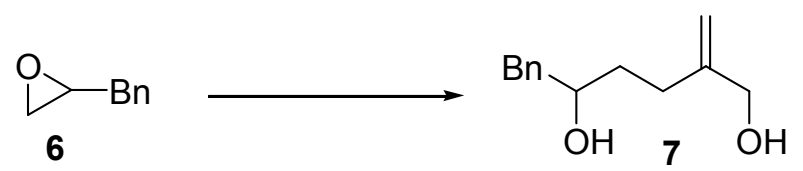

To a solution of Grignard (19 $\mathrm{mL}, 0.10 \mathrm{M}, 1.9 \mathrm{mmol})$ was added via cannula at RT a solution of epoxide $6(170 \mathrm{mg}, 1.26 \mathrm{mmol})$ in THF $(12.5 \mathrm{~mL})$ and the 
reaction stirred for $2 \mathrm{~h}$. The remaining organometallic reagent was quenched by the slow addition at $0^{\circ} \mathrm{C}$ of saturated aqueous $\mathrm{NH}_{4} \mathrm{Cl}$ solution. The product was extracted with ethyl acetate. The organic layer was washed with $1 \mathrm{~N} \mathrm{HCl}$, brine, dried over $\mathrm{MgSO}_{4}$ and concentrated in vacuo. The crude residue was purified by flash chromatography (60:40 petroleum ether/ethyl acetate), to give the desired product 7 as an oil (199 mg, 76\%); ${ }^{1} \mathrm{H}$ NMR (250 $\left.\mathrm{MHz}, \mathrm{CDCl}_{3}\right)$ : $\delta 7.37-$ $7.15(\mathrm{~m}, 5 \mathrm{H}, \mathrm{Ar}-\mathrm{H}), 5.02(\mathrm{br}, 1 \mathrm{H}$, alkene-H), 4.90 (br, 1H, alkene-H), 4.07 (s, $2 \mathrm{H}$, alkene- $\left.\mathrm{CH}_{2} \mathrm{OH}\right), 3.89-3.77(\mathrm{~m}, 1 \mathrm{H}, \mathrm{BnCH}), 2.82(\mathrm{dd}, J=13.5 \mathrm{~Hz}, J=4.5$ $\mathrm{Hz}, 1 \mathrm{H}, \mathrm{CH}_{2} \mathrm{Ph}$ ), 2.67 (dd, $J=13.5 \mathrm{~Hz}, J=8.0 \mathrm{~Hz}, 1 \mathrm{H}, \mathrm{CH}_{2} \mathrm{Ph}$ ), 2.37-2.05 (m, $2 \mathrm{H}$, alkene- $\left.\mathrm{CH}_{2} \mathrm{CH}_{2}\right)$, 2.01-1.81 (br, 2H, OH), 1.78-1.57 (m, 2H, alkene$\left.\mathrm{CH}_{2} \mathrm{CH}_{2}\right) ;{ }^{13} \mathrm{C}$ NMR $\left(62.9 \mathrm{MHz}, \mathrm{CDCl}_{3}\right): \delta$ 148.7, 138.5, 129.5, 128.7, 126.6, 110.1, 72.4, 66.1, 42.2, 34.8, 29.1; FTIR $\left(\mathrm{CH}_{2} \mathrm{Cl}_{2}\right)$ : 3436 (br), 2924 (s), 1652 (m), 1603 (m), 1495 (m), 1454 (s), 1082 (s), 1023 (s), 899 (m), 745 (m), 701 (s), 6047 (w), 529 (w) cm $\mathrm{cm}^{-1}$; HRMS (ES): $\mathrm{m} / \mathrm{z}[\mathrm{M}+\mathrm{Na}]^{+}$calcd for $\mathrm{C}_{13} \mathrm{H}_{18} \mathrm{O}_{2} \mathrm{Na}$ : 229.1204, found: 229.1196.

Preparation of 7-(benzyloxy)-2-methyleneheptane-1,5-diol (10).<smiles></smiles><smiles>C=C(CO)CCC(O)CCOBr</smiles>

To a solution of Grignard $(14.3 \mathrm{~mL}, 0.10 \mathrm{M}, 1.43 \mathrm{mmol})$ was added via cannula at RT a solution of epoxide $9(170 \mathrm{mg}, 0.95 \mathrm{mmol})$ in THF $(9.5 \mathrm{~mL})$ and the reaction stirred for $2 \mathrm{~h}$. The remaining organometallic reagent was quenched by the slow addition at $0^{\circ} \mathrm{C}$ of saturated aqueous $\mathrm{NH}_{4} \mathrm{Cl}$ solution. The product was extracted with ethyl acetate. The organic layer was washed with $1 \mathrm{~N} \mathrm{HCl}$, brine, dried over $\mathrm{MgSO}_{4}$ and concentrated in vacuo. The crude residue was purified by flash chromatography (60:40 petroleum ether/ethyl acetate), to give the desired product 10 as an oil (174 g, 74\%): ${ }^{1} \mathrm{H}$ NMR (250 MHz, $\left.\mathrm{CDCl}_{3}\right)$ : $\delta 7.47-$ $7.22(\mathrm{~m}, 5 \mathrm{H}, \mathrm{Ar}-\mathrm{H}), 5.01\left(\mathrm{br}, 1 \mathrm{H}\right.$, alkene- $\left.\mathrm{CH}_{2}\right), 4.87\left(\mathrm{br}, 1 \mathrm{H}\right.$, alkene- $\left.\mathrm{CH}_{2}\right), 4.51$ (s, $2 \mathrm{H}, \mathrm{OCH}_{2} \mathrm{Ph}$ ), 4.06 (br, 2H, alkene- $\mathrm{CH}_{2} \mathrm{OH}$ ), 3.95-3.77 (m, $1 \mathrm{H}, \mathrm{CH}_{2} \mathrm{CHOH}$ ), 3.77-3.58 (m, 2H, CH $\left.\mathrm{H}_{2} \mathrm{OBn}\right), 3.22(\mathrm{br}, 1 \mathrm{H}, \mathrm{OH}), 2.51-1.97\left(\mathrm{~m}, 3 \mathrm{H}, \mathrm{CH}_{2}, \mathrm{OH}\right)$, 1.88-1.50 (m, 4H, CH$) ;{ }^{13} \mathrm{C}$ NMR (62.9 MHz, $\left.\mathrm{CDCl}_{3}\right): \delta 149.0,138.0,128.6$, 127.9, 127.8, 109.8, 73.4, 71.1, 69.3, 66.1, 36.5, 35.6, 29.0; FTIR $\left(\mathrm{CH}_{2} \mathrm{Cl}_{2}\right)$ : 
3374 (br), 3088 (m), 3031 (m), 2920 (s), 2854 (s), 1652 (m), 1605 (w), 1586 (w), $1496(\mathrm{~m}), 1454(\mathrm{~m}), 1366(\mathrm{~m}), 1316(\mathrm{~m}), 1207(\mathrm{~m}), 1174(\mathrm{~m}), 1090(\mathrm{~m})$, 899 (m), 846 (w), 800 (w), 739 (m), 699 (m), 615 (w); HRMS (ES): m/z [M+Na] ${ }^{+}$ calcd for $\mathrm{C}_{15} \mathrm{H}_{22} \mathrm{O}_{3} \mathrm{Na}: 273.1467$, found: 273.1467 .

Preparation of (S)-6-(tert-butyldiphenylsilyloxy)-2-methylenehexane-1,5diol (13).

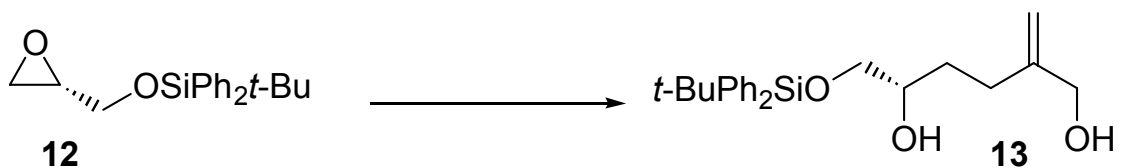

To a solution of Grignard $(17.6 \mathrm{~mL}, 0.085 \mathrm{M}, 1.5 \mathrm{mmol})$ was added via cannula at RT a solution of epoxide 12 (98\% ee, $312 \mathrm{mg}, 1.0 \mathrm{mmol})$ in THF (5 mL) and the reaction stirred for $2 \mathrm{~h}$. The remaining organometallic reagent was quenched by the slow addition at $0^{\circ} \mathrm{C}$ of saturated aqueous $\mathrm{NH}_{4} \mathrm{Cl}$ solution. The product was extracted with ethyl acetate. The organic layer was washed with $1 \mathrm{~N} \mathrm{HCl}$, brine, dried over $\mathrm{MgSO}_{4}$ and concentrated in vacuo. The crude residue was purified by flash chromatography (60:40 petroleum ether/ethyl acetate), to give the desired product 13 as an oil $(313 \mathrm{~g}, 82 \%): ;{ }^{1} \mathrm{H} \mathrm{NMR}\left(250 \mathrm{MHz}, \mathrm{CDCl}_{3}\right)$ : $\delta$ 7.78-7.54 (m, 4H, Ar-CH), 7.50-7.28 (m, 6H, Ar- $\mathrm{H}), 5.01$ (br, $1 \mathrm{H}$, alkene- $\mathrm{CH}_{2}$ ), $4.40\left(\mathrm{br}, 1 \mathrm{H}\right.$, alkene- $\mathrm{CH}_{2}$ ), $4.04\left(\mathrm{~d}, \mathrm{~J}=5.0 \mathrm{~Hz}, 2 \mathrm{H}\right.$, alkene- $\left.\mathrm{CH}_{2} \mathrm{OH}\right), 3.81-3.66$ (m, 1H, CHOH), 3.66 (dd, $\left.J=10.0 \mathrm{~Hz}, J=3.5 \mathrm{~Hz}, 1 \mathrm{H}, \mathrm{CH}_{2} \mathrm{OSi}\right), 3.51$ (dd, $J=$ 10.0 Hz, J = 3.5 Hz, 1H, CH $\left.3 \mathrm{H}, \mathrm{CH}_{2}, \mathrm{OH}\right), 1.70-1.49\left(\mathrm{~m}, 2 \mathrm{H}, \mathrm{CH}_{2}\right), 1.07$ (s, 9H, OSiPh $\left.2 \mathrm{C}\left(\mathrm{CH}_{3}\right)_{3}\right) ;{ }^{13} \mathrm{C} \mathrm{NMR}$ $\left(62.9 \mathrm{MHz}, \mathrm{CDCl}_{3}\right): \delta 148.7,135.6,133.2,130.0,127.9,109.9,71.7,68.0,66.0$, 30.9, 28.9, 27.0, 19.4; FTIR $\left(\mathrm{CH}_{2} \mathrm{Cl}_{2}\right)$ : 3356 (br), 3071 (m), 3050 (m), 2929 (s), 2857 (s), 1655 (w), $1472(\mathrm{~m}), 1428(\mathrm{~m}), 1391(\mathrm{w}), 1362(\mathrm{w}), 1308(\mathrm{w}), 1264(\mathrm{w})$, 1189 (w), 1113 (s), 1075 (s), 940 (w), 900 (m), 824 (m), 740 (m), 702 (s), 614 (m), 505 (s); HRMS (ES): $\mathrm{m} / \mathrm{z}[\mathrm{M}+\mathrm{Na}]^{+}$calcd for $\mathrm{C}_{23} \mathrm{H}_{32} \mathrm{O}_{3} \mathrm{NaSi}: 407.2018$, found: $407.2024 ;[\alpha]_{D}^{23}=-0.9\left(c=1.17\right.$ in $\left.\mathrm{CHCl}_{3}\right)$. 
<smiles>C1CCC2OC2C1</smiles>

15

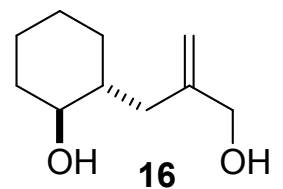

16

To a solution of Grignard ( $30 \mathrm{~mL}, 0.08 \mathrm{M}, 2.4 \mathrm{mmol}$ ) was added via cannula at RT a solution of epoxide $15(162 \mu \mathrm{l}, 1.6 \mathrm{mmol})$ in THF (4 mL) and the reaction stirred for $2 \mathrm{~h}$. The remaining organometallic reagent was quenched by the slow addition at $0^{\circ} \mathrm{C}$ of saturated aqueous $\mathrm{NH}_{4} \mathrm{Cl}$ solution. The product was extracted with ethyl acetate. The organic layer was washed with $1 \mathrm{~N} \mathrm{HCl}$, brine, dried over $\mathrm{MgSO}_{4}$ and concentrated in vacuo. The crude residue was purified by flash chromatography (60:40 petroleum ether/ethyl acetate), to give the desired product 16 as a white solid (136 mg, 50\%); ${ }^{1} \mathrm{H}$ NMR $\left(250 \mathrm{MHz}, \mathrm{CDCl}_{3}\right)$ : $\delta 5.03\left(\mathrm{br}, 1 \mathrm{H}\right.$, alkene- $\left.\mathrm{CH}_{2}\right), 4.87\left(\mathrm{br}, 1 \mathrm{H}\right.$, alkene- $\left.\mathrm{CH}_{2}\right), 4.09(\mathrm{~d}, J=5.0 \mathrm{~Hz}, 2 \mathrm{H}$, alkene- $\left.\mathrm{CH}_{2} \mathrm{OH}\right), 3.31-3.16(\mathrm{~m} 1 \mathrm{H}$, cyclohexane- $\mathrm{CHOH}), 2.58-2.51(\mathrm{~m}, 3 \mathrm{H}$, cyclohexane- $\mathrm{CH}$, and $\left.\mathrm{CH}_{2}\right), 2.00-1.56\left(\mathrm{~m}, 5 \mathrm{H}, \mathrm{CH}_{2}, \mathrm{OH}\right), 1.50-0.78(\mathrm{~m}, 5 \mathrm{H}$, $\left.\mathrm{CH}_{2}\right) ;{ }^{13} \mathrm{C} \mathrm{NMR}\left(62.9 \mathrm{MHz}, \mathrm{CDCl}_{3}\right): \delta 148.1,111.8,75.5,66.0,43.7,37.2,35.8$, 31.1, 25.6, 25.2; FTIR $\left(\mathrm{CH}_{2} \mathrm{Cl}_{2}\right)$ : 3329 (br), 2927 (s), 2855 (s), 1654 (w), 1448 (m), $1355(w), 1310(w), 1236(w), 1133(w), 1104(w), 1060(m), 1034(s), 928$ $(w), 897(m), 852(w), 827(w), 668(w), 563(w)$; HRMS (EI): m/z [M] ${ }^{+}$calcd for $\mathrm{C}_{10} \mathrm{H}_{18} \mathrm{O}_{2}: 170.1307$, found: 170.1306 ; $\mathrm{Mp}: 73-74^{\circ} \mathrm{C}$.

Preparation of 4-methylene-1-phenylpentane-1,5-diol (19a) and 2methylene-4-phenylpentane-1,5-diol (19b).

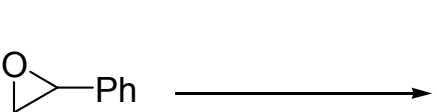

18<smiles>C=C(CO)CCC(O)c1ccccc1</smiles><smiles>C=C(CO)CC(CO)c1ccccc1</smiles>

To a solution of Grignard $(21.2 \mathrm{~mL}, 0.10 \mathrm{M}, 2.12 \mathrm{mmol})$ was added via cannula at RT a solution of epoxide $18(170 \mathrm{mg}, 1.41 \mathrm{mmol})$ in THF $(14 \mathrm{~mL})$ and the reaction stirred for $2 \mathrm{~h}$. The remaining organometallic reagent was quenched by the slow addition at $0^{\circ} \mathrm{C}$ of saturated aqueous $\mathrm{NH}_{4} \mathrm{Cl}$ solution. The product was extracted with ethyl acetate. The organic layer was washed with $1 \mathrm{~N} \mathrm{HCl}$, brine, dried over $\mathrm{MgSO}_{4}$ and concentrated in vacuo. The crude residue was purified by flash chromatography (60:40 petroleum ether/ethyl acetate), to give the 
desired products as yellow oils: 19b (165 mg, 61\%) and 19a (55 mg, 20\%); 19b; ${ }^{1} \mathrm{H}$ NMR (250 MHz, $\left.\mathrm{CDCl}_{3}\right): \delta 7.31-7.05(\mathrm{~m}, 5 \mathrm{H}, \mathrm{Ar}-\mathrm{H}), 4.87(\mathrm{br}, 1 \mathrm{H}$, alkene- $H), 4.69(\mathrm{br}, 1 \mathrm{H}$, alkene- $H), 3.85\left(\mathrm{br}, 2 \mathrm{H}\right.$, alkene- $\left.\mathrm{CH}_{2} \mathrm{OH}\right), 3.6(\mathrm{~d}, J=6.5$ $\left.\mathrm{Hz}, 2 \mathrm{H}, \mathrm{CH}(\mathrm{Ph}) \mathrm{CH}_{2} \mathrm{OH}\right), 2.61(\mathrm{~m}, 3 \mathrm{H}, \mathrm{CHPh}$, and $\mathrm{OH}), 2.49(\mathrm{dd}, 1 \mathrm{H}, J=14.0$ $\left.\mathrm{Hz}, J=6.0 \mathrm{CH}_{2}\right), 2.24\left(\mathrm{dd}, 1 \mathrm{H}, J=14.0 \mathrm{~Hz}, J=6.5 \mathrm{~Hz}, \mathrm{CH}_{2}\right) ;{ }^{13} \mathrm{C} \operatorname{NMR}(62.9$ $\mathrm{MHz}_{\mathrm{CDCl}}$ ): $\delta 146.7,142.3,128.9,128.3,127.1,112.5,67.4,66.2,47.0,35.8$; FTIR ( $\left.\mathrm{CH}_{2} \mathrm{Cl}_{2}\right)$ : 3335 (br), 3085 (m), 3029 (m), 2926 (s), 2874 (s),1651 (m), 1602 (m), 1583 (w), $1494(\mathrm{~m}), 1453$ (s), 1337 (m), $1312(\mathrm{~m}), 1211(\mathrm{~m}), 1062$ (s), 1026 (s), 901 (s), 840 (w), 759 (s), 701 (s), 572 (m), 538 (m); HRMS (El): $\mathrm{m} / \mathrm{z}\left[\mathrm{M}-\mathrm{H}_{2} \mathrm{O}\right]^{+}$calcd for $\mathrm{C}_{12} \mathrm{H}_{14} \mathrm{O}: 174.1044$, found: 174.1049 , and $19 a,{ }^{1} \mathrm{H}$ NMR (250 MHz, $\left.\mathrm{CDCl}_{3}\right): \delta$ 7.42-7.20 (m, 5H, Ar-H), 5.06 (br, 1H, alkene-H), 4.91 (br, $1 \mathrm{H}$, alkene- $H$ ), $4.68(\mathrm{dd}, J=7.5 \mathrm{~Hz}, J=5.5 \mathrm{~Hz}, 1 \mathrm{H}, \mathrm{PhCH}), 4.05(\mathrm{~s}, 2 \mathrm{H}$, alkene$\left.\mathrm{CH}_{2} \mathrm{OH}\right), 2.41-1.77\left(\mathrm{~m}, 6 \mathrm{H}, \mathrm{CH}_{2}, \mathrm{OH}\right) ;{ }^{13} \mathrm{C} \mathrm{NMR}\left(62.9 \mathrm{MHz}, \mathrm{CDCl}_{3}\right): \delta$ 148.5, 144.7, 128.6, 127.6, 126.0, 110.0, 74.1, 66.0, 37.1, 29.1; FTIR $\left(\mathrm{CH}_{2} \mathrm{Cl}_{2}\right): 3336$ (br), 2923 (s),1653 (m), $1494(\mathrm{~m}), 1453$ (s), $1311(\mathrm{~m}), 1267$ (m), $1204(\mathrm{~m})$, 1062 (s), 1026 (s), 901 (m), 764 (m), 701 (m), 630 (w), 544 (w); HRMS (El): m/z $[\mathrm{M}]^{+}$calcd for $\mathrm{C}_{12} \mathrm{H}_{16} \mathrm{O}_{2}:$ 192.1150, found: 192.1153 .

\section{Palladium catalyzed cyclisation}

Preparation 2-benzyl-5-methylenetetrahydro-2H-pyran (8).

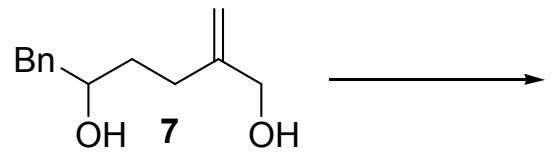<smiles>C=C1CCC(Br)OC1</smiles>

A round bottom flask with molecular sieves $4 \AA$ (500 $\mathrm{mg})$ was flame dried under vacuo and placed under a nitrogen atmosphere. To this was added palladium acetate (34 mg, $0.15 \mathrm{mmol}$ ), triphenylphosphine (159 mg, $0.12 \mathrm{mmol}$ ) and anhydrous toluene $(7 \mathrm{~mL})$. The suspension was stirred for $10 \mathrm{~min}$, and a solution of allylic alcohol 7 (625 $\mathrm{mg}, 3.03 \mathrm{mmol})$ in anhydrous toluene $(23 \mathrm{~mL})$ was transferred via cannula to the reaction mixture. Titanium ispropoxide (224 $\mu \mathrm{L}, 0.76 \mathrm{mmol}$ ) was added and the reaction mixture was heated at reflux for 1.5 h. After cooling to RT, direct purification by chromatography on silica gel (gradient starting with petroleum ether and ending with 98:2 petroleum ether/EtOAc) provided the pyran 8 as an oil (420 mg, 82\%); ${ }^{1} \mathrm{H}$ NMR (250 MHz, 
$\left.\mathrm{CDCl}_{3}\right): \delta$ 7.41-7.12 (m, 5H, Ar-H), 4.83-4.73 (m, 2H, alkene-CH$H_{2}, 4.18(\mathrm{~d}, J=$ $12.0 \mathrm{~Hz}, 1 \mathrm{H}$, pyran-OCH ), $3.94\left(\mathrm{~d}, \mathrm{~J}=12.0 \mathrm{~Hz}, 1 \mathrm{H}\right.$, pyran-OCH $\left.\mathrm{CH}_{2}\right), 3.70-3.55$ (m, 1H, pyran-OCH), $2.91\left(\mathrm{dd}, J=14.0 \mathrm{~Hz}, J=7.0 \mathrm{~Hz}, \mathrm{CH}_{2} \mathrm{Ph}\right), 2.66$ (dd, $J=$ $\left.14.0 \mathrm{~Hz}, J=6.0 \mathrm{~Hz}, \mathrm{CH}_{2} \mathrm{Ph}\right), 2.47-2.33\left(\mathrm{~m}, 1 \mathrm{H}, \mathrm{CH}_{2}\right), 2.29-2.12\left(\mathrm{~m}, 1 \mathrm{H}, \mathrm{CH}_{2}\right)$, 1.82-1.70 (m, $\left.1 \mathrm{H}, \mathrm{CH}_{2}\right), 1.53-1.33\left(\mathrm{~m}, 1 \mathrm{H}, \mathrm{CH}_{2}\right) ;{ }^{13} \mathrm{C}$ NMR $\left(62.9 \mathrm{MHz}, \mathrm{CDCl}_{3}\right): \delta$ 143.9, 138.8, 129.5, 128.4, 126.3, 109.3, 78.4, 72.6, 42.7, 33.0, 31.6; FTIR $\left(\mathrm{CH}_{2} \mathrm{Cl}_{2}\right): 3066(\mathrm{~m}), 3028(\mathrm{~m}), 2940$ (s), $2834(\mathrm{~s}), 1654(\mathrm{w}), 1605(\mathrm{w}), 1496(\mathrm{~m})$, $1454(\mathrm{~m}), 1437(\mathrm{~m}), 1371(\mathrm{~m}), 1339(\mathrm{~m}), 1287$ (w), $1250(\mathrm{w}), 1210(\mathrm{w}), 1080$ (s), $1029(\mathrm{~m}), 899$ (s) cm $\mathrm{cm}^{-1}$; HRMS (El): m/z [M] calcd for $\mathrm{C}_{13} \mathrm{H}_{16} \mathrm{O}: 188.1201$, found: 188.1208 .

\section{Preparation of 2-(2-(benzyloxy)ethyl)-5-methylenetetrahydro-2H-pyran (11)}<smiles>[R]OCC(=C)CCC(O)CCOBr</smiles><smiles>C=C1CCC(CCOc2ccccc2)OC1</smiles>

Following the representative procedure above, a solution of allylic alcohol 10 (150 $\mathrm{mg}, 0.60 \mathrm{mmol})$ in anhydrous toluene $(7 \mathrm{~mL})$ was transferred via cannula to the suspension (molecular sieves $4 \AA$ (150 mg), $\mathrm{Pd}(\mathrm{OAc})_{2}(6.7 \mathrm{mg}, 0.03$ $\mathrm{mmol}), \mathrm{PPh}_{3}(31.5 \mathrm{mg}, 0.12 \mathrm{mmol})$ and anhydrous toluene $\left.(2 \mathrm{~mL})\right)$. Titanium ispropoxide $(43.0 \mu \mathrm{L}, 0.15 \mathrm{mmol})$ was added and the reaction mixture was heated at reflux for $1.5 \mathrm{~h}$. After cooling to RT, direct purification by chromatography on silica gel (gradient starting with petroleum ether and ending with 98:2 petroleum ether/EtOAc) provided the desired product 11 as an oil (80 mg, 85\%); ${ }^{1} \mathrm{H}$ NMR (250 MHz, $\mathrm{CDCl}_{3}$ ): $\delta$ 7.39-7.23 (m, 5H, Ar-H), 4.81-4.73 (m, $2 \mathrm{H}$, alkene- $\mathrm{CH}_{2}$ ), 4.51 (s, $2 \mathrm{H}, \mathrm{OCH}_{2} \mathrm{Bn}$ ), 4.16 (dd, $J=12.5 \mathrm{~Hz}, J=1.5 \mathrm{~Hz}, 1 \mathrm{H}$,

pyran- $\mathrm{OCH}_{2}$ ), 3.95 (d, $J=12.5 \mathrm{~Hz}, 1 \mathrm{H}$, pyran-OCH ), 3.67-3.51 (m, 3H, pyran$\mathrm{OCH}$, and $\left.\mathrm{CH}_{2} \mathrm{OBn}\right), 2.46-2.35\left(\mathrm{~m}, 1 \mathrm{H}, \mathrm{CH}_{2}\right), 2.34-2.18\left(\mathrm{~m}, 1 \mathrm{H}, \mathrm{CH}_{2}\right), 1.84-1.70$ $\left(\mathrm{m}, 3 \mathrm{H}, \mathrm{CH}_{2}\right), 1.50-1.32\left(\mathrm{~m}, 1 \mathrm{H}, \mathrm{CH}_{2}\right) ;{ }^{13} \mathrm{C}$ NMR $\left(62.9 \mathrm{MHz}, \mathrm{CDCl}_{3}\right): \delta$ 144.1, 138.7, 128.5, 127.7, 127.6, 109.2, 74.4, 73.1, 72.5, 67.0, 36.3, 33.6, 31.7; FTIR $\left(\mathrm{CH}_{2} \mathrm{Cl}_{2}\right): 3069(\mathrm{~m}), 3030(\mathrm{~m}), 2941(\mathrm{~s}), 2853(\mathrm{~s}), 1656(\mathrm{~m}), 1496(\mathrm{~m}), 1453$ (m), $1441(\mathrm{~m}), 1364(\mathrm{~m}), 1339(\mathrm{~m}), 1206(\mathrm{~m}), 1083(\mathrm{~s}), 1053(\mathrm{~s}), 1028(\mathrm{~m}), 900$ 
(m), $884(\mathrm{~m}), 736(\mathrm{~m}), 698(\mathrm{~m}), 670(\mathrm{w}), 612(\mathrm{w}) \mathrm{cm}^{-1}$; HRMS (El): m/z [M] ${ }^{+}$ calcd for $\mathrm{C}_{15} \mathrm{H}_{20} \mathrm{O}_{2}: 232.1463$, found: 232.1472 .

Preparation of (S)-tert-butyl((5-methylenetetrahydro-2H-pyran-2yl)methoxy)diphenylsilane (14)

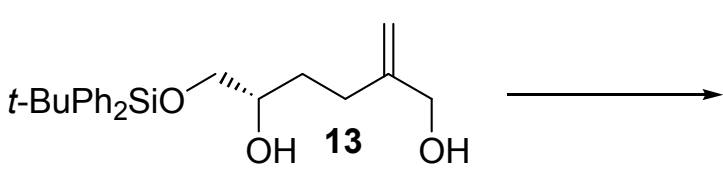

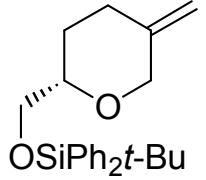

14

Following the representative procedure above, a solution of allylic alcohol 13 $(1.04 \mathrm{~g}, 2.70 \mathrm{mmol})$ in toluene $(25 \mathrm{~mL})$ was transferred via cannula to the suspension (molecular sieves $4 \AA$ (450 mg), $\mathrm{Pd}(\mathrm{OAc})_{2}(30.4 \mathrm{mg}, 0.13 \mathrm{mmol})$, $\mathrm{PPh}_{3}(142 \mathrm{mg}, 0.54 \mathrm{mmol})$ in anhydrous toluene $\left.(10 \mathrm{~mL})\right)$. Titanium ispropoxide (200 $\mu \mathrm{L}, 0.68 \mathrm{mmol}$ ) was added and the reaction mixture was heated at reflux for $1.5 \mathrm{~h}$. After cooling to RT, direct purification by chromatography on silica gel (gradient starting with petroleum ether and ending with 98:2 petroleum ether/EtOAc) provided the desired product 14 as an oil (820 mg, 83\%, 98\% ee) The enantiomeric purity of pyran 14 was determined by chiral HPLC analysis (Chiral Technologies Chiralpak AD, RT, 99.5:0.5 hexane/isopropanol, 1.0 $\mathrm{mL} / \mathrm{min}, t_{\mathrm{R}}($ minor $)=5.38 \mathrm{~min}, t_{\mathrm{R}}$ (major) $\left.=4.52 \mathrm{~min}\right) ;{ }^{1} \mathrm{H} \mathrm{NMR}(250 \mathrm{MHz}$, $\left.\mathrm{CDCl}_{3}\right)$ : $\delta$ 7.72-7.63 (m, 4H, Ar-H), 7.47-7.32 (m, 6H, Ar-H), 4.81-4.75 (m, 2H, alkene- $\mathrm{CH}_{2}$ ), $4.18\left(\mathrm{dd}, J=12.5 \mathrm{~Hz}, J=1.5 \mathrm{~Hz}, 1 \mathrm{H}\right.$, pyran-OCH $\left.\mathrm{H}_{2}\right), 3.96(\mathrm{~d}, J=$ $12.5 \mathrm{~Hz}, 1 \mathrm{H}$, pyran- $\left.\mathrm{OCH}_{2}\right), 3.82-3.70(\mathrm{~m}, 1 \mathrm{H}$, pyran- $\mathrm{OCH}), 3.62-3.48(\mathrm{~m}, 2 \mathrm{H}$, $\left.\mathrm{CH}_{2} \mathrm{OSi}\right), 2.50-2.38\left(\mathrm{~m}, 1 \mathrm{H}, \mathrm{CH}_{2}\right), 2.35-2.18\left(\mathrm{~m}, 1 \mathrm{H}, \mathrm{CH}_{2}\right), 1.96-1.84(\mathrm{~m}, 1 \mathrm{H}$, $\left.\mathrm{CH}_{2}\right), 1.49-1.29\left(\mathrm{~m}, 1 \mathrm{H}, \mathrm{CH}_{2}\right), 1.06\left(\mathrm{~s}, 9 \mathrm{H}, \mathrm{OSiPh}_{2} \mathrm{C}\left(\mathrm{CH}_{3}\right)_{3}\right) ;{ }^{13} \mathrm{C}$ NMR $(62.9$ $\left.\mathrm{MHz}_{\mathrm{CDCl}}\right): \delta 144.2,135.8,133.8,129.7,127.8,109.3,77.8,72.3,67.1,31.3$, 30.2, 27.0, 19.4; FTIR ( $\left.\mathrm{CH}_{2} \mathrm{Cl}_{2}\right)$ : 3072 (s), 3050 (s), 2999 (s), 2932 (s), 2854 (s), $1657(\mathrm{~m}), 1590(\mathrm{~m}), 1472(\mathrm{~m}), 1428(\mathrm{~m}), 1390(\mathrm{~m}), 1362(\mathrm{~m}), 1338(\mathrm{w}), 1325$ (w), $1287(w), 1263(w), 1240(w), 1204(w), 1187(m), 1135(m), 1113(m)$, $1083(\mathrm{~m}), 1047$ (m), 1008 (m), 969 (w), 938 (w), 901 (m), $824(\mathrm{~m}), 807$ (m), 740 (m), $702(\mathrm{~m}), 614(\mathrm{~m}), \mathrm{cm}^{-1}$; HRMS (ES): m/z [M+Na] ${ }^{+}$calcd for $\mathrm{C}_{23} \mathrm{H}_{30} \mathrm{O}_{2} \mathrm{NaSi}$ : 389.1913, found: $389.1928 ;[\alpha]_{D}^{22}=-4.8\left(c=1.16\right.$ in $\left.\mathrm{CHCl}_{3}\right)$ 
Preparation 3-methyleneoctahydro-2H-chromene (17)

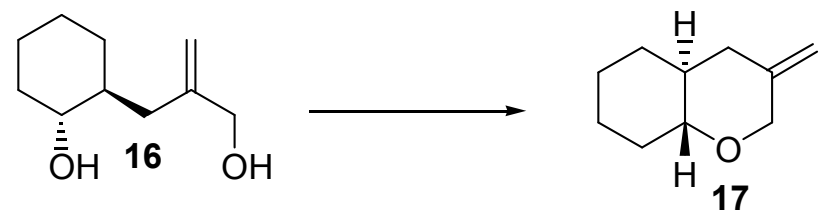

Following the representative procedure above, a solution of allylic alcohol 16 (100 $\mathrm{mg}, 0.59 \mathrm{mmol}$ ) in anhydrous toluene $(4 \mathrm{~mL}$ ) was transferred via cannula to the suspension (molecular sieves $4 \AA$ ( $80 \mathrm{mg}$ ), $\mathrm{Pd}(\mathrm{OAc})_{2}(6.6 \mathrm{mg}, 0.029$ $\mathrm{mmol}), \mathrm{PPh}_{3}(30.9 \mathrm{mg}, 0.12 \mathrm{mmol})$ and anhydrous toluene $\left.(1.5 \mathrm{~mL})\right)$. Titanium ispropoxide ( $44 \mu \mathrm{L}, 0.68 \mathrm{mmol}$ ) was added and the reaction mixture was heated at reflux for $1.5 \mathrm{~h}$. After cooling to RT, direct purification by chromatography on silica gel (gradient starting with petroleum ether and ending with 98:2 petroleum ether/EtOAc) provided the desired product 17 as an oil (60 $\mathrm{mg}, 67 \%)$. The compound showed satisfactory spectra data ${ }^{\mathrm{v}} ;{ }^{1} \mathrm{H}$ NMR $(250$ $\left.\mathrm{MHz}, \mathrm{CDCl}_{3}\right): \delta 4.88-4.63\left(\mathrm{~m}, 2 \mathrm{H}\right.$, alkene- $\left.\mathrm{CH}_{2}\right), 4.21(\mathrm{dd}, J=12.0 \mathrm{~Hz}, J=2.0$ $\mathrm{Hz}, 1 \mathrm{H}$, pyran-OCH $\left.\mathrm{O}_{2}\right), 3.97\left(\mathrm{~d}, J=12.0 \mathrm{~Hz}, 1 \mathrm{H}\right.$, pyran-OCH $\left.\mathrm{C}_{2}\right), 3.10-2.90(\mathrm{~m}, 1 \mathrm{H}$, pyran-OCH), 2.32 (ddd, $J=13.5 \mathrm{~Hz}, J=4.0 \mathrm{~Hz}, J=1.5 \mathrm{~Hz}, 1 \mathrm{H}$, alkene$\left.\left(\mathrm{CH}_{2}\right) \mathrm{CH}\right), 1.96-1.74\left(\mathrm{~m}, 3 \mathrm{H}\right.$, alkene- $\left(\mathrm{CH}_{2}\right) \mathrm{CH}$, and $\left.\mathrm{CH}_{2}\right), 1.70-1.63(\mathrm{~m}, 2 \mathrm{H}$, $\left.\mathrm{CH}_{2}\right)$, 1.43-1.13 (m, 4H, CH$), 1.11-0.96\left(\mathrm{~m}, 1 \mathrm{H}\right.$, alkene- $\left.\mathrm{CH}_{2} \mathrm{CH}\right) ;{ }^{13} \mathrm{C} \mathrm{NMR}$ $\left(62.9 \mathrm{MHz}, \mathrm{CDCl}_{3}\right): \delta 144.5,109.1,81.5,72.6,43.9,39.1,32.4,32.1,25.6$, 25.1; HRMS (El): $\mathrm{m} / \mathrm{z}[\mathrm{M}]^{+}$calcd for $\mathrm{C}_{10} \mathrm{H}_{16} \mathrm{O}: 152.1201$, found: 152.1201.

Preparation 5-methylene-2-phenyltetrahydro-2H-pyran (20a).

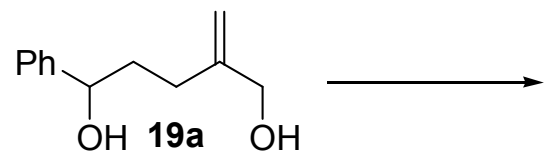<smiles>C=C1CCC(c2ccccc2)OC1</smiles>

Following the representative procedure above, a solution of diol 19a (195 mg, $1.01 \mathrm{mmol})$ in toluene $(8.5 \mathrm{~mL})$ was transferred via cannula to the suspension (molecular sieves $4 \AA$ (150 mg), $\mathrm{Pd}(\mathrm{OAc})_{2}$ (11.3 mg, $0.05 \mathrm{mmol}$ ), $\mathrm{PPh}_{3}$ (53 mg, $0.20 \mathrm{mmol}$ ) and anhydrous toluene $1.5 \mathrm{~mL})$ ). Titanium ispropoxide $(75 \mu \mathrm{L}, 0.25$ $\mathrm{mmol}$ ) was added and the reaction mixture was heated at toluene reflux for 1.5 h. After cooling to RT, direct purification by chromatography on silica gel (gradient starting with petroleum ether and ending with 98:2 petroleum 
ether/EtOAc) provided the desired product 20a as an oil $(116 \mathrm{mg}, 66 \%):{ }^{1} \mathrm{H}$ NMR (250 MHz, $\left.\mathrm{CDCl}_{3}\right)$ : $\delta$ 7.40-7.22 (m, 5H, Ar-H), 4.90-4.80 (m, 2H, alkene$\left.\mathrm{CH}_{2}\right), 4.47(\mathrm{dd}, \mathrm{J}=11.0 \mathrm{~Hz}, \mathrm{~J}=2.0 \mathrm{~Hz}, 1 \mathrm{H}$, pyran-OCH), $4.36(\mathrm{dd}, J=12.5 \mathrm{~Hz}$, $J=1.5 \mathrm{~Hz}, 1 \mathrm{H}$, pyran-OCH$\left.H_{2}\right), 4.16\left(\mathrm{~d}, J=12.5 \mathrm{~Hz}, 1 \mathrm{H}\right.$, pyran-OCH $\left.\mathrm{H}_{2}\right), 2.60-2.37$ (m, 2H, alkene- $\left.\mathrm{CH}_{2}\right), 2.05-1.93\left(\mathrm{~m}, 1 \mathrm{H}, \mathrm{CH}_{2} \mathrm{CHPh}\right), 1.84-1.65(\mathrm{~m}, 1 \mathrm{H}$, alkene$\left.\mathrm{CH}_{2} \mathrm{CH}_{2}\right) ;{ }^{13} \mathrm{C}$ NMR $\left(62.9 \mathrm{MHz}, \mathrm{CDCl}_{3}\right): \delta 143.5,142.5,128.4,127.6,126.0$, 109.7, 79.7, 72.9, 35.5, 32.2; FTIR $\left(\mathrm{CH}_{2} \mathrm{Cl}_{2}\right): 3069$ (w), 3030 (w), 2942 (s), 2831 (s), $1495(w), 1452(\mathrm{~m}), 1439(\mathrm{w}), 1357$ (w), 1337 (w), $1285(\mathrm{w}), 1244(\mathrm{w}), 1089$ (s), 1070 (s), 1028 (s), 902 (s), 754 (m), 699 (s) cm ; HRMS (EI): m/z [M] calcd for $\mathrm{C}_{12} \mathrm{H}_{14} \mathrm{O}: 174.1045$, found: 174.1051 .

\section{Preparation 3-methylene-5-phenyltetrahydro-2H-pyran (20b)}

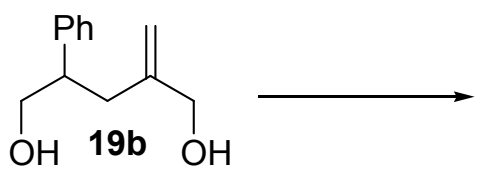<smiles>C=C1CO[14C](=O)CC(c2ccccc2)C1</smiles>

Following the representative procedure above, a solution of allylic alcohol $19 \mathrm{~b}$ (75 $\mathrm{mg}, 0.39 \mathrm{mmol})$ in toluene $(3.5 \mathrm{~mL}$ ) was transferred via cannula to the suspension (molecular sieves $4 \AA$ (60 mg), $\mathrm{Pd}(\mathrm{OAc})_{2}(4.4 \mathrm{mg}, 0.019 \mathrm{mmol})$, $\mathrm{PPh}_{3}(20.4 \mathrm{mg}, 0.078 \mathrm{mmol})$ and anhydrous toluene $\left.1.0 \mathrm{~mL}\right)$ ). Titanium ispropoxide $(75 \mu \mathrm{L}, 0.25 \mathrm{mmol})$ was added and the reaction mixture was heated at toluene reflux for $1.5 \mathrm{~h}$. After cooling to RT, direct purification by chromatography on silica gel (gradient starting with petroleum ether and ending with 98:2 petroleum ether/EtOAc) provided the desired product $\mathbf{2 0 b}$ as an oil (54 mg, 79\%): ${ }^{1} \mathrm{H}$ NMR (250 MHz, $\mathrm{CDCl}_{3}$ ): $\delta$ 7.40-7.18 (m, 5H, Ar-H), 4.95-4.78 $(\mathrm{m}, 2 \mathrm{H}$, alkene- $H), 4.87\left(\mathrm{~d}, J=12.0 \mathrm{~Hz}, 1 \mathrm{H}\right.$, alkene- $\left.\mathrm{CH}_{2}\right), 4.23(\mathrm{~d}, J=12.0 \mathrm{~Hz}$, $1 \mathrm{H}$, pyran-OCH), 4.08-3.95 (m, 2H, pyran-OCH$)_{2}, 3.52(\mathrm{t}, J=10.5 \mathrm{~Hz}, 1 \mathrm{H}$, pyran-OCH$H_{2}$, 3.07-2.90 (m, 1H, CHPh), 2.72-2.60 (m, 1H, CH $\left.\mathrm{H}_{2} \mathrm{CHPh}\right), 2.57-$ $2.42\left(\mathrm{~m}, 1 \mathrm{H}\right.$, alkene- $\left(\mathrm{CH}_{2}\right) \mathrm{CHPh} ;{ }^{13} \mathrm{C} \mathrm{NMR}\left(62.9 \mathrm{MHz}, \mathrm{CDCl}_{3}\right): \delta$ 143.1, 141.7, 128.7, 127.4, 126.9, 110.3, 73.2, 72.3, 44.6, 38.7; FTIR $\left(\mathrm{CH}_{2} \mathrm{Cl}_{2}\right): 3072(\mathrm{~m})$, 3029 (m), 2957 (s), 2901 (s), 2835 (s), 1656 (m), 1603 (m), 1494 (s), 1453 (s), $1436(\mathrm{~m}), 1337$ (w), $1281(\mathrm{w}), 1252(\mathrm{w}), 1199(\mathrm{w}), 1174(\mathrm{~m}), 1085(\mathrm{~s}), 1030(\mathrm{w})$, $984(\mathrm{~m}), 932(\mathrm{~m}), 905(\mathrm{~s}), 871(\mathrm{~m}), 756(\mathrm{~s}), 700(\mathrm{~s}), 654(\mathrm{~m}), 524(\mathrm{~m}) \mathrm{cm}^{-1}$; HRMS (El): m/z [M] calcd for $\mathrm{C}_{12} \mathrm{H}_{14} \mathrm{O}: 174.1045$, found: 174.1048. 


\section{(+)-Rhopaloic Acid B}

Preparation of (S)-(2-(oxiran-2-yl)ethoxy)(tert-butyl)diphenylsilane (21)
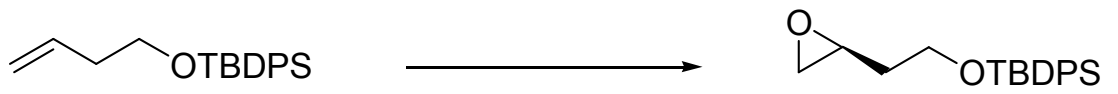

21

To a solution of tert-butyl(but-3-enyloxy)diphenylsilane vi $(8.76 \mathrm{~g}, 28.20 \mathrm{mmol})$ in DCM $(65 \mathrm{~mL})$ at $0^{\circ} \mathrm{C}$ was added portionwise $\mathrm{m}$-CPBA $(12.6 \mathrm{~g}, 56.40 \mathrm{~mol})$. The resulting suspension was warmed gradually to temperature, and stirred for $20 \mathrm{~h}$. The reaction mixture was filtered to remove the white precipitate, concentrated in vacuo, and re-dissolved in petroleum ether. The suspension was filtered, the filtrate concentrated in vacuo and purified by flash chromatography (90:10 petroleum ether/ethyl acetate), to give the racemic epoxide (8.80 g, $95 \%)$ as pale yellow oil.

The kinetic resolution of epoxide $\mathbf{2 1}$ was conducted following the procedure of Jacobsen. $^{\text {vii }}$ The catalyst $(S, S)$-salen-Co $(145 \mathrm{mg}, 0.24 \mathrm{mmol})$ was dissolved in DCM $(3.1 \mathrm{ml})$ and treated with acetic acid $(136 \mu \mathrm{L}, 2.40 \mathrm{mmol})$. The solution was allowed to stir at RT open to air for 30 min over which time the colour changed from orange-red to dark brown. The solution was concentrated in vacuo to leave a crude brown solid. The resulting catalyst residue was dissolved in THF (1.6 mL), and then racemic epoxide 21 (7.8 g, $23.90 \mathrm{mmol})$ was added to the solution. The reaction was cooled at $0^{\circ} \mathrm{C}$ and $\mathrm{H}_{2} \mathrm{O}(236 \mu \mathrm{L}$, $13.14 \mathrm{mmol}$ ) was added dropwise over a $5 \mathrm{~min}$ period. The reaction was allowed to warm to RT and stirred for 40h. Direct purification by flash chromatography on silica gel (90:10 petroleum ether/ethyl acetate) provided the enantioenriched epoxide 21 (3.45 g, 44\%, >99\% ee). The enantiomeric purity of epoxide 21 was determined by chiral HPLC analysis (Chiral Technologies Chiralpak OD, RT, 98:2 hexane/isopropanol, $1.0 \mathrm{~mL} / \mathrm{min}, t_{\mathrm{R}}$ (minor) $=5.34 \mathrm{~min}$, $t_{\mathrm{R}}$ (major) $\left.=6.09 \mathrm{~min}\right) .[\alpha]_{\mathrm{D}}^{23}=-4.7\left(\mathrm{c}=1.06, \mathrm{CHCl}_{3}\right)$; lit. ${ }^{\text {viii }}[\alpha]_{\mathrm{D}}{ }^{18}=-6.0(\mathrm{c}=$ 8.49, $\left.\mathrm{CHCl}_{3}\right) ;{ }^{1} \mathrm{H}$ NMR (250 MHz, $\left.\mathrm{CDCl}_{3}\right): \delta$ 7.73-7.60 (m, 4H, Ar-H), 7.44-7.33 $(\mathrm{m}, 6 \mathrm{H}, \mathrm{Ar}-\mathrm{H}), 3.90-3.74\left(\mathrm{~m}, 2 \mathrm{H}, \mathrm{CH}_{2} \mathrm{OSi}\right), 3.14-3.06(\mathrm{~m}, 1 \mathrm{H}$, epoxide-CHO$)$, $2.78\left(\mathrm{dd}, J=5.0 \mathrm{~Hz}, J=4.0 \mathrm{~Hz} 1 \mathrm{H}\right.$, epoxide- $\mathrm{CH}_{2} \mathrm{O}$ ), $2.51(\mathrm{dd}, J=5.0 \mathrm{~Hz}, J=$ $2.5 \mathrm{~Hz} 1 \mathrm{H}$, epoxide- $\mathrm{CH}_{2} \mathrm{O}$ ), 1.82-1.72 (m, 2H, $\left.\mathrm{CH}_{2} \mathrm{CH}_{2} \mathrm{OSi}\right), 1.05$ (s, 9H, $\mathrm{CH}_{3}$ ); 
${ }^{13} \mathrm{C}$ NMR $\left(62.9 \mathrm{MHz}, \mathrm{CDCl}_{3}\right): \delta 135.6,133.8,139.8,127.8,61.0,50.2,47.3$, $35.8,26.9,19.3$.

Preparation of (R)-7-(tert-butyldiphenylsilyloxy)-2-methyleneheptane-1,5diol (22).

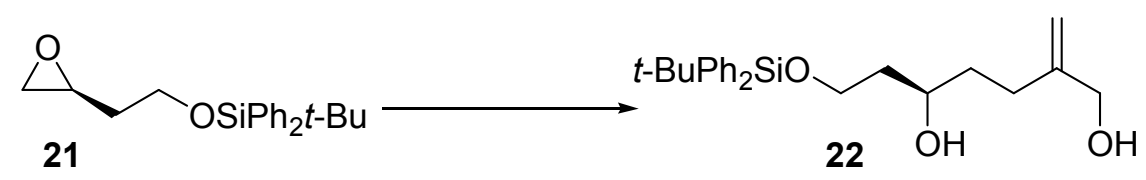

To a solution of Grignard (10 mL, 0.14M, $1.4 \mathrm{mmol})$ was added via cannula at RT a solution of epoxide 21 (304 mg, $0.93 \mathrm{mmol})$ in THF (9.5 mL) and the reaction stirred for $2 \mathrm{~h}$. The remaining organometallic reagent was quenched by the slow addition at $0^{\circ} \mathrm{C}$ of saturated aqueous $\mathrm{NH}_{4} \mathrm{Cl}$ solution. The product was extracted with ethyl acetate. The organic layer was washed with $1 \mathrm{~N} \mathrm{HCl}$, brine, dried over $\mathrm{MgSO}_{4}$ and concentrated in vacuo. The crude residue was purified by flash chromatography (60:40 petroleum ether/ethyl acetate), to give the desired product 22 as an oil (293 mg, 79\%); ${ }^{1} \mathrm{H}$ NMR (250 MHz, $\left.\mathrm{CDCl}_{3}\right): \delta 7.70-$ $7.63(\mathrm{~m}, 4 \mathrm{H}, \mathrm{Ar}-\mathrm{H}), 7.48-7.33(\mathrm{~m}, 6 \mathrm{H}, \mathrm{Ar}-\mathrm{H})$, 5.06-5.01 (m, $1 \mathrm{H}$, alkene- $H)$, 4.92$4.88(\mathrm{~m}, 1 \mathrm{H}$, alkene- $\mathrm{H}), 4.09\left(\mathrm{br}, 2 \mathrm{H}\right.$, alkene- $\left.\mathrm{CH}_{2} \mathrm{OH}\right), 3.99-3.78(\mathrm{~m}, 3 \mathrm{H}, \mathrm{CHOH}$ and $\left.\mathrm{CH}_{2} \mathrm{OSi}\right), 3.50(\mathrm{br}, 1 \mathrm{H}, \mathrm{OH}), 2.33-1.94\left(\mathrm{~m}, 3 \mathrm{H}\right.$, alkene- $\mathrm{CH}_{2} \mathrm{CH}_{2}$ and $\left.\mathrm{OH}\right)$, 1.84-1.54 (m, 4H, CH (62.9 MHz, $\left.\mathrm{CDCl}_{3}\right)$ : $\delta 149.0,135.7,133.1$, 133.0, 130.0, 127.9, 109.7, 71.5, 66.1, 63.6, 38.5, 35.7, 29.0, 26.9, 19.1; IR (film, cm-1): 3354 (br), 3071 (w), 3050 (w), 2932 (s), 2858 (s), 1652 (w), 1472 (m), 1428 (s), 1390 (m), 1361 (m), 1112 (s), 1085 (s), $1029(\mathrm{~m}), 900(\mathrm{~m}), 823(\mathrm{~m}), 737(\mathrm{~m}), 702(\mathrm{~s}), 688(\mathrm{~m}), 614$ (m), 504 (s); HRMS (ES): m/z [M+H] ${ }^{+}$calcd for $\mathrm{C}_{24} \mathrm{H}_{35} \mathrm{O}_{3} \mathrm{Si}: 399.2355$, found: 399.2371; $[\alpha]_{D}^{23}=+14.4\left(\mathrm{c}=1.04\right.$ in $\left.\mathrm{CHCl}_{3}\right)$.

Preparation of (2-((R)-tetrahydro-5-methylene-2H-pyran-2-yl)ethoxy)(tertbutyl)diphenylsilane (23)
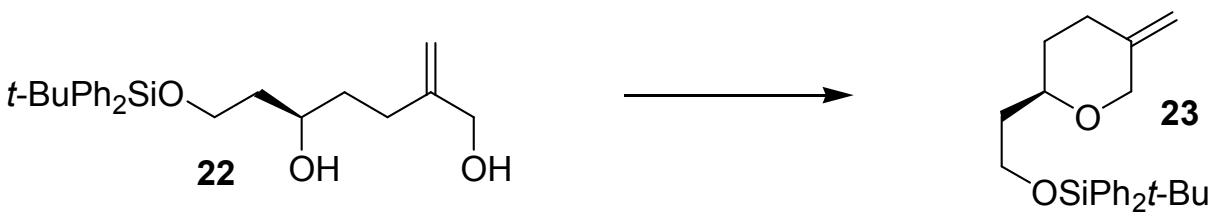
Following the representative procedure above, a solution of 22 (1.12 g, 2.81 $\mathrm{mmol}$ ) in toluene (42 $\mathrm{mL})$ was transferred via cannula to the suspension

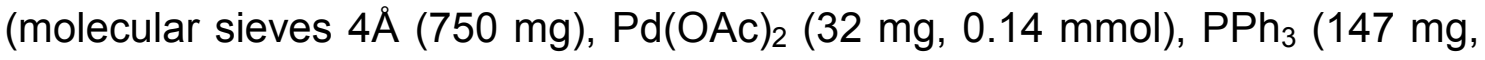
$0.56 \mathrm{mmol}$ ) and anhydrous toluene $15 \mathrm{~mL})$ ). Titanium ispropoxide (208 $\mu \mathrm{L}, 0.70$ $\mathrm{mmol}$ ) was added and the reaction mixture was heated at toluene reflux for 1.5 h. After cooling to RT, direct purification by chromatography on silica gel (gradient starting with petroleum ether and ending with 98:2 petroleum ether/EtOAc) provided the desired product as an oil (920 mg, 86\%, >99\% ee). The enantiomeric purity of pyran 23 was determined by chiral HPLC analysis (Chiral Technologies Chiralpak AD, RT, 99.5:0.5 hexane/isopropanol, 1.0 $\mathrm{mL} / \mathrm{min}, t_{\mathrm{R}}$ (minor) $=3.86 \mathrm{~min}, t_{\mathrm{R}}$ (major) $\left.=4.22 \mathrm{~min}\right) ;{ }^{1} \mathrm{H}$ NMR $(250 \mathrm{MHz}$, $\left.\mathrm{CDCl}_{3}\right)$ : $\delta$ 7.72-7.62 (m, 4H, Ar-H), 7.46-7.32 (m, 6H, Ar-H), 4.79-4.73 (m, 2H, alkene- $\mathrm{CH}_{2}$ ), $4.13(\mathrm{dd}, J=12.5 \mathrm{~Hz}, J=2.0 \mathrm{~Hz}, 1 \mathrm{H}$, pyran-OCH$), 3.92(\mathrm{~d}, J=$ $12.5 \mathrm{~Hz}, 1 \mathrm{H}$, pyran-OCH $\left.\mathrm{C}_{2}\right), 3.89-3.70\left(\mathrm{~m}, 2 \mathrm{H}, \mathrm{CH}_{2} \mathrm{OSi}\right), 3.69-3.56(\mathrm{~m}, 1 \mathrm{H}$, pyran-OCH), 2.46-2.34 (m, $\left.1 \mathrm{H}, \mathrm{CH}_{2}\right), 2.33-2.16\left(\mathrm{~m}, 1 \mathrm{H}, \mathrm{CH}_{2}\right), 1.83-1.67(\mathrm{~m}, 3 \mathrm{H}$, $\left.\mathrm{CH}_{2}\right), 1.48-1.28\left(\mathrm{~m}, 1 \mathrm{H}, \mathrm{CH}_{2}\right), 1.05\left(\mathrm{~s}, 9 \mathrm{H}, \mathrm{OSiPh}_{2}\left(\mathrm{CH}_{3}\right)_{3}\right) ;{ }^{13} \mathrm{C} \mathrm{NMR}(100 \mathrm{MHz}$, $\left.\mathrm{CDCl}_{3}\right): \delta 144.2,135.7,134.2,134.1,129.7,127.7,109.2,74.1,72.4,60.5$, 39.0, 33.6, 31.8, 27.0, 19.4; IR (film, cm $\mathrm{cm}^{-1}$ ): 3049 (w), 2932 (s), 2857 (s), 1472 (m), $1428(\mathrm{~m}), 1389(\mathrm{w}), 1361(\mathrm{w}), 1263$ (w), $1112(\mathrm{~s}), 1086(\mathrm{~s}), 944(\mathrm{w}), 900$ (m), $823(\mathrm{~m}), 737(\mathrm{~m}), 702(\mathrm{~s}), 614(\mathrm{w}), 505(\mathrm{~m})$; HRMS (ES): m/z [M+Na] ${ }^{+}$ calcd for $\mathrm{C}_{24} \mathrm{H}_{32} \mathrm{O}_{2} \mathrm{NaSi}: 403.2069$, found: 403.2064, $[\alpha]_{\mathrm{D}}{ }^{22}=-5.5$ (c = 1.27, $\left.\mathrm{CHCl}_{3}\right)$.

Preparation of cis-, trans-(6-(2-(tert-butyldiphenylsilyloxy)ethyl)tetrahydro2H-pyran-3-yl)methanol (24)

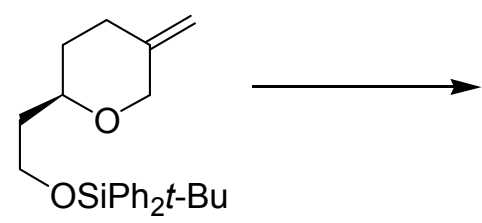

23

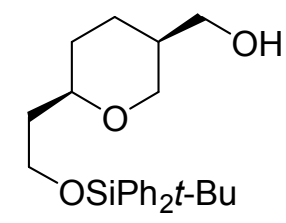

cis-24

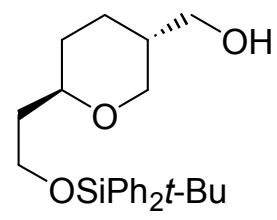

trans-24

To a solution of 23 (958 $\mathrm{mg}, 2.52 \mathrm{mmol})$ in anhydrous THF (25 mL) and $\mathrm{RhCl}\left(\mathrm{PPh}_{3}\right)_{3} \quad(117 \mathrm{mg}, \quad 0.126 \mathrm{mmol})$ at $-20^{\circ} \mathrm{C}$, was added dropwise catecholborane $^{\mathrm{ix}}(940 \mu \mathrm{L}, 8.82 \mathrm{mmol})$. The reaction was stirred for $20 \mathrm{~h}$ at $-20^{\circ} \mathrm{C}$ 
and then quenched with $\mathrm{NaOH}(1 \mathrm{M}, 10.1 \mathrm{~mL}, 10.08 \mathrm{mmol}), \mathrm{H}_{2} \mathrm{O}_{2}(1.16 \mathrm{~mL}$, $10.08 \mathrm{mmol}$ ), and stirred for $3 \mathrm{~h}$ at RT. After this time, saturated aqueous $\mathrm{NH}_{4} \mathrm{Cl}$ solution was added to the reaction and the product extracted with EtOAc. The combined organic extracts were washed with saturated aqueous $\mathrm{K}_{2} \mathrm{CO}_{3}$ solution, brine, dried over $\mathrm{MgSO}_{4}$ and concentrated in vacuo. The crude residue was purified by flash chromatography (60:40 petroleum ether/ethyl acetate), to give a mixture of the desired products 24 as yellow oil (876 $\mathrm{mg}$, $88 \%, 1: 3$ trans/cis ratio); $\delta$ 7.80-7.57 (m, 4H, Ar- $\mathrm{H}$, (trans + cis)), 7.52-7.28 (m, 6H, Ar-H, (trans + cis)), $4.06(\mathrm{ddd}, J=11.0 \mathrm{~Hz}, J=4.0 \mathrm{~Hz}, J=2.5 \mathrm{~Hz}, 0.25$ $\mathrm{H}$ (trans), pyran-OCH), 3.95 (d, $J=12.0 \mathrm{~Hz}, 0.75 \mathrm{H}$ (cis), pyran- $\mathrm{OCH}_{2}$ ), 3.89$3.33\left(\mathrm{~m}, 5.75 \mathrm{H}, \mathrm{CH}_{2} \mathrm{OSi}\right.$, pyran- $\mathrm{OCH}_{2}$, pyran-OCH, $\mathrm{CH}_{2} \mathrm{OH}$ (trans + cis)), 3.13 (t, $J=11.0 \mathrm{~Hz}, 0.25$ (trans), pyran-OCH$\left.{ }_{2}\right)$ 2.43-1.15 (m, 8H, CH$, \mathrm{CH}, \mathrm{OH}$ (trans + cis)), $1.06\left(\mathrm{~s}, 9 \mathrm{H},-\mathrm{OSiPh}_{2} \mathrm{C}\left(\mathrm{CH}_{3}\right)_{3}\right) ;{ }^{13} \mathrm{C} \mathrm{NMR}\left(62.9 \mathrm{MHz}, \mathrm{CDCl}_{3}\right): \delta$ 135.7, 134.2, 134.1, 129.6, 127.7, 74.8, 74.7 (trans), 71.0 (trans), 68.5, 65.1 (trans), 63.4, 60.5, 60.3 (trans), 39.2 (trans), 39.1, 36.0, 31.5 (trans), 27.9 , 27.0, 24.6, 19.4; IR (film, cm ${ }^{-1}$ ): 3382 (br), 3071 (w), 3050 (w), 2931 (s), 2857 (s), $1472(\mathrm{~m}), 1428(\mathrm{~m}), 1112(\mathrm{~s}), 1091(\mathrm{~s}), 823(\mathrm{~m}), 737(\mathrm{~m}), 702(\mathrm{~s}), 614(\mathrm{~m})$, 505 (m) HRMS (ES): m/z [M+Na] ${ }^{+}$calcd for $\mathrm{C}_{24} \mathrm{H}_{34} \mathrm{O}_{2} \mathrm{NaSi}: 421.2175$, found: 421.2177.

Preparation of cis-, trans-tert-butyl(2-(5-(iodomethyl)tetrahydro-2H-pyran2-yl)ethoxy)diphenylsilane (25)

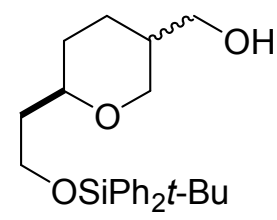

24

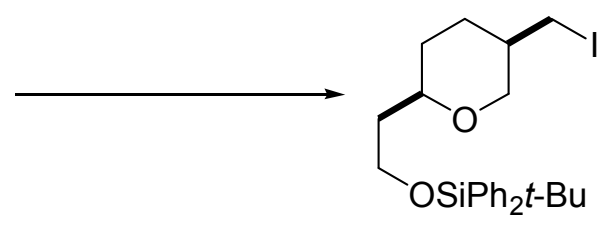

cis-25

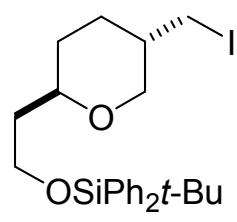

Trans-25

A cis/trans mixture (3:1) of 24 from (876 $\mathrm{mg}, 2.19 \mathrm{mmol}$ ) was dissolved in anhydrous THF (44 mL). Imidazole (300 mg, $4.40 \mathrm{mmol})$, triphenylphosphine (866 mg, $3.29 \mathrm{mmol}$ ), iodine (837 $\mathrm{mg}, 3.29 \mathrm{mmol}$ ) were added to the solution at $R T$. The reaction was stirred for $3 h$ at $R T$, and then quenched with $\mathrm{H}_{2} \mathrm{O}$ and EtOAc. The aqueous layer was extracted with EtOAc, and the combined 
organic extracts were washed with saturated aqueous $\mathrm{NaHSO}_{3}$ solution, aqueous $1 \mathrm{~N} \mathrm{HCl}$ solution, saturated aqueous $\mathrm{NaHCO}_{3}$ solution, brine, dried over $\mathrm{MgSO}_{4}$ and concentrated in vacuo. The crude residue was purified by flash chromatography (99:1; petroleum ether/EtOAc), to give the desired products as yellow oil; cis-25 (765 mg, 68\%) and of trans-25 (258 mg, 23\%): (trans-25) ${ }^{1} \mathrm{H}$ NMR (250 MHz, $\left.\mathrm{CDCl}_{3}\right): \delta$ 7.72-7.63 (m, 4H, Ar-H), 7.48-7.33 (m, $6 \mathrm{H}, \mathrm{Ar}-\mathrm{H}$ ), 4.09 (ddd, $J=11.0 \mathrm{~Hz}, J=4.0 \mathrm{~Hz}, J=2.5 \mathrm{~Hz}, 1 \mathrm{H}, \mathrm{CH}_{2}-\mathrm{O}$ ), 3.89-3.68 (m, 2H, CH $\mathrm{CHSi}_{2}, 3.50-3.38(\mathrm{~m}, 1 \mathrm{H}, \mathrm{CH}-\mathrm{O}), 3.09-2.91\left(\mathrm{~m}, 3 \mathrm{H}, \mathrm{CH}_{2} \mathrm{O}, \mathrm{CH}_{2} \mathrm{l}\right)$ 2.06-1.95 (m, $\left.1 \mathrm{H}, \mathrm{CHCH}_{2} \mathrm{l}\right), 1.84-1.62\left(\mathrm{~m}, 4 \mathrm{H}, \mathrm{CH}_{2}\right), 1.42-1.10\left(\mathrm{~m}, 2 \mathrm{H}, \mathrm{CH}_{2}\right)$, $1.06\left(\mathrm{~s}, 9 \mathrm{H}, \mathrm{CH}_{3}\right) ;{ }^{13} \mathrm{C}$ NMR $\left(100 \mathrm{MHz} \mathrm{CDCl}_{3}\right): \delta 135.9,134.3,134.2,129.9$, 127.9, 74.8, 73.4, 60.6, 39.1, 38.3, 31.7, 31.4, 27.2, 19.6, 8.4; IR (film, cm${ }^{-1}$ ): 3070 (w), 3048 (w), 2931 (s), 2856 (s), 1472 (m), 1461 (w), 1428 (m), 1389 (w), $1360(w), 1297(w), 1261(w), 1233(w), 1188(m), 1184(m), 1146(w), 1112(s)$, 1090 (s), 1015 (w), 941 (w), 907 (w), $823(\mathrm{~m}), 737(\mathrm{~m}), 702(\mathrm{~s}), 688(\mathrm{~m}), 614$ (m), 504 (s), 488 (m); HRMS (ES): m/z [M+Na] ${ }^{+}$calcd for $\mathrm{C}_{24} \mathrm{H}_{33} \mathrm{O}_{2} \mathrm{INaSi}$ : 531.1192, found: $531.1183,[\alpha]_{\mathrm{D}}{ }^{22}=+3.4^{\circ}\left(\mathrm{c}=1.19\right.$ in $\left.\mathrm{CHCl}_{3}\right)$; $\left(\right.$ cis-25) ${ }^{1} \mathrm{H}$ NMR (250 MHz, $\left.\mathrm{CDCl}_{3}\right): \delta$ 7.71-7.63 (m, 4H, Ar-H), 7.48-7.33 (m, 6H, Ar-H), 3.93 (dt, $\left.J=12.0 \mathrm{~Hz}, J=2.0 \mathrm{~Hz}, 1 \mathrm{H}, \mathrm{CH}_{2}-\mathrm{O}\right), 3.88-3.67\left(\mathrm{~m}, 2 \mathrm{H}, \mathrm{CH}_{2}-\mathrm{O}\right.$ and $\left.\mathrm{CH}-\mathrm{O}\right), 3.64-$ $3.42\left(\mathrm{~m}, 3 \mathrm{H}, \mathrm{CH}_{2} \mathrm{OSi}\right.$ and $\left.\mathrm{CH}_{2} \mathrm{l}\right), 3.31\left(\mathrm{dd}, 1 \mathrm{H}, J=9.5 \mathrm{~Hz}, J=7.0 \mathrm{~Hz}, 1 \mathrm{H}, \mathrm{CH}_{2} \mathrm{l}\right)$, 2.00-1.57 (m, 5H, CH$), 1.48-1.25\left(\mathrm{~m}, 2 \mathrm{H}, \mathrm{CH}_{2}\right.$ and $\left.\mathrm{CHCH}_{2} \mathrm{l}\right), 1.06\left(\mathrm{~s}, 9 \mathrm{H}, \mathrm{CH}_{3}\right)$ $;{ }^{13} \mathrm{C} \mathrm{NMR}\left(100 \mathrm{MHz}, \mathrm{CDCl}_{3}\right): \delta 135.7,134.1,134.0,129.7,127.7,74.6,70.4$, 60.5, 39.0, 37.4, 28.2, 27.2, 27.1, 19.6, 9.9; IR (film, $\mathrm{cm}^{-1}$ ): 3070 (w), 3048 (w), 2931 (s), 2856 (s), 1472 (m), 1446 (w), 1428 (m), 1389 (w), 1360 (w), 1295 (w), 1271 (w), 1207 (w), 1177 (w), 1112 (s), 1090 (s), 1024 (m), 1007 (w), 969 (w), $940(\mathrm{w}), 906$ (w), $823(\mathrm{~m}), 800(\mathrm{w}), 736(\mathrm{~m}), 703(\mathrm{~s}), 614(\mathrm{~m}), 504(\mathrm{~s}), 490(\mathrm{~m})$; HRMS (ES): $\mathrm{m} / \mathrm{z}[\mathrm{M}+\mathrm{H}]^{+}$calcd for $\mathrm{C}_{24} \mathrm{H}_{34} \mathrm{IO}_{2}: 509.1373$, found: $509.1355,[\alpha]_{D}{ }^{22}$ $=+8.6\left(\mathrm{c}=1.04\right.$ in $\left.\mathrm{CHCl}_{3}\right)$.

Preparation of 1-((2E,6E)-3,7,11-trimethyldodeca-2,6,10trienylsulfonyl)benzene (26).
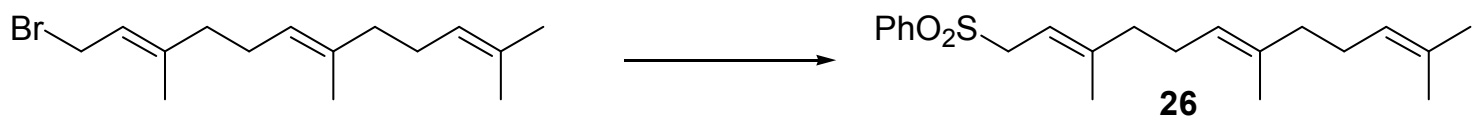
Sodium benzenesulfonic acid (581 $\mathrm{mg}, 3.54 \mathrm{mmol}$ ) and tetra-butyl-ammonium iodide (16 mg, $0.04 \mathrm{mmol}$ ) were dissolved in anhydrous DMF (22mL). After stirring for $10 \mathrm{~min}$, farnesyl bromide $(800 \mu \mathrm{L}, 2.95 \mathrm{mmol})$ was added dropwise and the reaction mixture was stirred at RT for $3.5 \mathrm{~h}$. The reaction was quenched with $\mathrm{H}_{2} \mathrm{O}$ and $\mathrm{Et}_{2} \mathrm{O}$ and the aqueous layer was extracted two times with $\mathrm{Et}_{2} \mathrm{O}$. The combined organic layers were washed three times with $\mathrm{H}_{2} \mathrm{O}$, brine, dried over $\mathrm{Na}_{2} \mathrm{SO}_{4}$ and concentrated in vacuo. The crude residue was purified by flash chromatography (90:10 petroleum ether/ethyl acetate), to give the desired product 26 as a yellow oil (900 mg, 88\%). The compound showed satisfactory spectra data ${ }^{\mathrm{x}}{ }^{1}{ }^{\mathrm{H}} \mathrm{NMR}\left(250 \mathrm{MHz}, \mathrm{CDCl}_{3}\right)$ : $\delta$ 7.90-7.83 (m, $\left.2 \mathrm{H}, \mathrm{Ar}-\mathrm{H}\right)$, 7.67-7.47 $(\mathrm{m}, 3 \mathrm{H}, \mathrm{Ar}-\mathrm{H}), 5.18$ (td, $\mathrm{J}=7.9 \mathrm{~Hz}, J=1.2 \mathrm{~Hz}, 1 \mathrm{H}$, alkene- $\mathrm{H}-\mathrm{CH}_{2} \mathrm{SO}_{2} \mathrm{Ph}$ ), 5.12$5.00(\mathrm{~m}, 2 \mathrm{H}$, alkene- $\mathrm{H}), 3.79\left(\mathrm{~d}, \mathrm{~J}=7.9 \mathrm{~Hz}, 2 \mathrm{H}, \mathrm{CH}_{2} \mathrm{SO}_{2} \mathrm{Ph}\right), 2.19-1.89(\mathrm{~m}, 8 \mathrm{H}$, $\left.\mathrm{CH}_{2}\right), 1.66\left(\mathrm{~d}, \mathrm{~J}=1.0 \mathrm{~Hz}, 3 \mathrm{H}, \mathrm{PhSO}_{2} \mathrm{CH}_{2}\right.$-alkene- $\left.\mathrm{CH}_{3}\right), 1.61-1.56\left(\mathrm{~m}, 6 \mathrm{H}, \mathrm{CH}_{3}\right)$, $1.30\left(\mathrm{~d}, J=1.2 \mathrm{~Hz}, 3 \mathrm{H}, \mathrm{CH}_{3}\right) ;{ }^{13} \mathrm{C} \operatorname{NMR}\left(63 \mathrm{MHz}, \mathrm{CDCl}_{3}\right): \delta 146.4,138.7$, $135.7,133.5,131.4,128.9,128.5,124.2$, 123.3, 110.3, 56.1, 39.7, 26.7, 26.2, 25.7, 17.7, 16.2, 16.0 .

Preparation of tert-butyldiphenyl(2-((2R,5R)-5-((3E,7E)-4,8,12trimethyltrideca-3,7,11-trienyl)tetrahydro-2H-pyran-2-yl)ethoxy)silane (28)

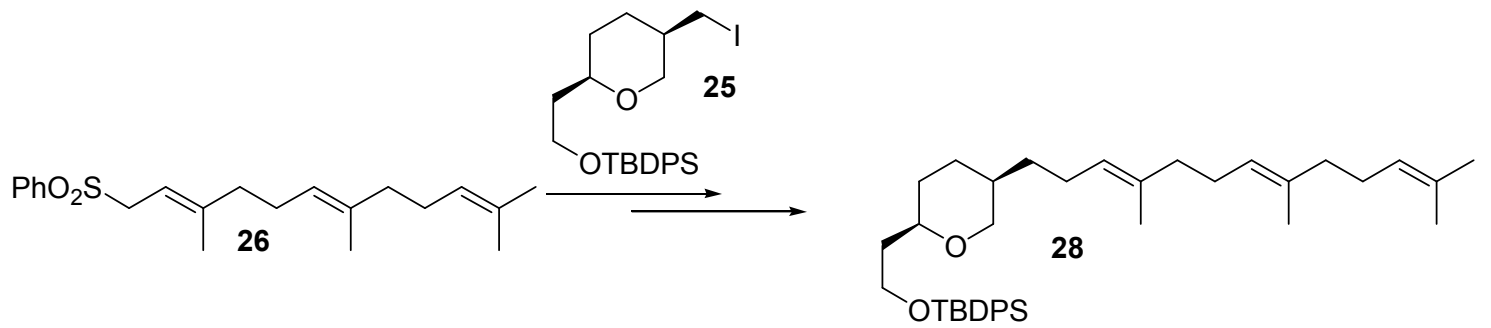

LDA $(200 \mu \mathrm{L}$ of DIPA, $710 \mu \mathrm{L}$ of $1.9 \mathrm{M} \mathrm{n}-\mathrm{BuLi}, 1.45 \mathrm{~mL}$ THF) was added to a cooled $\left(-78^{\circ} \mathrm{C}\right)$ solution of the farnesyl sulfone $26(131 \mathrm{mg}, 0.378 \mathrm{mmol})$ in anhydrous THF $(1.4 \mathrm{~mL})$ and DMPU $(520 \mu \mathrm{L})$. The resulting bright orange solution was stirred for $45-60$ min at $-78^{\circ} \mathrm{C}$, at which point a solution of pyran 25 (160 $\mathrm{mg}, 0.315 \mathrm{mmol})$ in anhydrous THF $(0.5 \mathrm{~mL})$, was added dropwise. The reaction was stirred an additional $1 \mathrm{~h}$ at $-78^{\circ} \mathrm{C}$, and then the cold bath was removed and the reaction stirred for another $3-4 \mathrm{~h}$. The reaction mixture was then quenched with a saturated solution of ammonium chloride. The aqueous solution was extracted three times with ether, dried over magnesium sulphate, 
and concentrated in vacuo. The resulting oil residue was purified via flash chromatography on silica gel (petroleum ether/ethyl acetate 90:10) to give an inseparable mixture of the desired product $\mathbf{2 7}$ and farnesyl sulfone $\mathbf{2 5}$.

The crude mixture of sulfone $(248 \mathrm{mg}$ ) was dissolved in anhydrous THF (3.8 $\mathrm{mL}$ ) and $\mathrm{PdCl}_{2}$ (dppf) (27.7 $\mathrm{mg}, 0.04 \mathrm{mmol}$, based on the farnesyl sulfone) was added. Super Hydride (1M in THF, $1.13 \mathrm{~mL}, 1.13 \mathrm{mmol}$ based on the farnesyl sulfone) was added dropwise at $10^{\circ} \mathrm{C}$ to the orange solution which quickly changed to red-brown. The cold bath was removed and the reaction was stirred at room temperature for $6 \mathrm{~h}$, at which time it was quenched carefully at $0^{\circ} \mathrm{C}$ with saturated aqueous $\mathrm{NH}_{4} \mathrm{Cl}$ solution. The resulting aqueous layer was extracted with ether three times. The combined organic layers were washed with brine, dried over $\mathrm{MgSO}_{4}$, filtered, and concentrated in vacuo. The resulting oily residue was purified via flash chromatography on silica gel (98:2; petroleum ether/EtOAc) to give the desired product 28 (135 $\mathrm{mg}, 73 \%$ over two steps) as a yellow oil. ${ }^{1} \mathrm{H}$ NMR (250 MHz, $\left.\mathrm{CDCl}_{3}\right)$ : $\delta$ 7.75-7.66 (m, 4H, Ar-H), 7.46-7.35 (m, $6 \mathrm{H}, \mathrm{Ar}-\mathrm{H}), 5.20-4.08(\mathrm{~m}, 3 \mathrm{H}$, alkene- $\mathrm{H}), 3.91-3.69\left(\mathrm{~m}, 3 \mathrm{H}, \mathrm{CH}_{2} \mathrm{O}\right.$ and $\left.\mathrm{CH}-\mathrm{O}\right)$, 3.65-3.51 (m, 2H, CH $\left.\mathrm{H}_{2} \mathrm{OSi}\right), 2.10-1.80\left(\mathrm{~m}, 10 \mathrm{H}, \mathrm{CH}_{2}\right), 1.89-1.59(\mathrm{~m}, 21 \mathrm{H}$, alkene- $\left.\left.\mathrm{CH}_{3}, \mathrm{CH}_{2}, \mathrm{CH}\right), 1.09\left(\mathrm{~s}, 9 \mathrm{H}, \mathrm{CH}_{3}\right) ;{ }^{13} \mathrm{C} \mathrm{NMR} \mathrm{(62.9} \mathrm{MHz,} \mathrm{CDCl} 3\right)$ : $\delta$ $135.7,135.1$, 135.0, 134.2, 134.1, 131.3, 129.6, 127.7, 124.6, 124.5, 124.4, 74.5, 70.8, 60.6, 39.9 (2C), 38.8, 33.4, 30.6, 27.6, 27.5, 27.0, 26.9, 26.7, 25.9, 19.4, 17.8, 16.2 (2C); IR (film, cm ${ }^{-1}$ ): 3071 (s), 3050 (s), 2928 (s, br), 2738 (m), 1472 (s), 1428 (s), 1384 (s), 1360 (s), 1260 (m), 1206 (m), 1189 (m), 1112 (s, br), 1007 (m), 939 (m), 900 (w), 824 (s), 736 (s), 702 (s), 614 (s), 504 (s); HRMS (ES): $\mathrm{m} / \mathrm{z}[\mathrm{M}+\mathrm{H}]^{+}$calcd for $\mathrm{C}_{39} \mathrm{H}_{59} \mathrm{O}_{2} \mathrm{Si}: 587.4284$, found: 587.4285 , $[\alpha]_{D}^{22}=+7.4\left(\mathrm{c}=1.08\right.$ in $\left.\mathrm{CHCl}_{3}\right)$.

\section{Preparation of 2-((2R,5R)-tetrahydro-5-((3E,7E)-4,8,12-trimethyltrideca-} 3,7,11-trienyl)-2H-pyran-2-yl)ethanol (29)

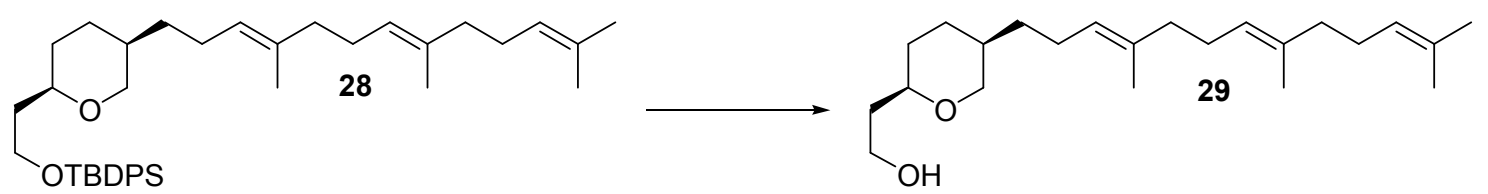

To a solution of 28 (143 mg, $0.24 \mathrm{mmol})$ in anhydrous THF (2.5 mL) was added dropwise TBAF (1M in THF, $370 \mu \mathrm{L}, 0.37 \mathrm{mmol}$ ). The reaction was stirred at RT 
for $20 \mathrm{~h}$. The reaction was quenched with $\mathrm{H}_{2} \mathrm{O}$. The aqueous layer was extracted two times with EtOAc. The combined organic extracts were washed with brine, dried over $\mathrm{MgSO}_{4}$ and concentrated in vacuo. The crude residue was purified by flash chromatography (80:20 petroleum ether/ethyl acetate), to give the desired product 29 as colourless oil $(84 \mathrm{~g}, 98 \%):{ }^{1} \mathrm{H}$ NMR (250 MHz, $\left.\mathrm{CDCl}_{3}\right): \delta$ 5.14-4.99 (m, 3H, alkene- $\left.\mathrm{H}\right), 3.80-3.67\left(\mathrm{~m}, 3 \mathrm{H}, \mathrm{CH}_{2} \mathrm{O}\right.$ and $\left.\mathrm{CH}-\mathrm{O}\right)$, 3.56-3.45 (m, 2H, CH $\left.\mathrm{H}_{2} \mathrm{OH}\right), 2.94(\mathrm{~s}, \mathrm{br}, 1 \mathrm{H}, \mathrm{OH}), \quad 2.12-1.87\left(\mathrm{~m}, 10 \mathrm{H}, \mathrm{CH}_{2}\right)$, 1.82-1.31 (m, 21H, alkene- $\left.\left.\mathrm{CH}_{3}, \mathrm{CH}_{2}, \mathrm{CH}\right) ;{ }^{13} \mathrm{C} \mathrm{NMR} \mathrm{(62.9} \mathrm{MHz,} \mathrm{CDCl}_{3}\right)$ : $\delta$ 135.2, 135.0, 131.3, 124.4 (2C), 124.3, 78.4, 71.0, 61.5, 39.8 (2C), 37.8, 33.0, $30.2,27.4,27.2,26.8,26.7,25.9,25.8,17.8,16.0$ (2C); IR (film, cm ${ }^{-1}$ ): 3396 (br), 2928 (s), 2854 (s), 1450 (s), 1381 (s), 1201 (w), 1110 (m), 1065 (s), 897 (w), 863 (w), 834 (w), 741 (w), 604 (w), 538 (w), 490 (w); HRMS (ES): m/z $[\mathrm{M}+\mathrm{H}]^{+}$calcd for $\mathrm{C}_{23} \mathrm{H}_{41} \mathrm{O}_{2}: 349.3107$, found: $349.3113,[\alpha]_{D}^{22}=+13.9(c=1.01$, $\left.\mathrm{CHCl}_{3}\right)$.

\section{Preparation of (+)-Rhopaloic Acid B (30)}

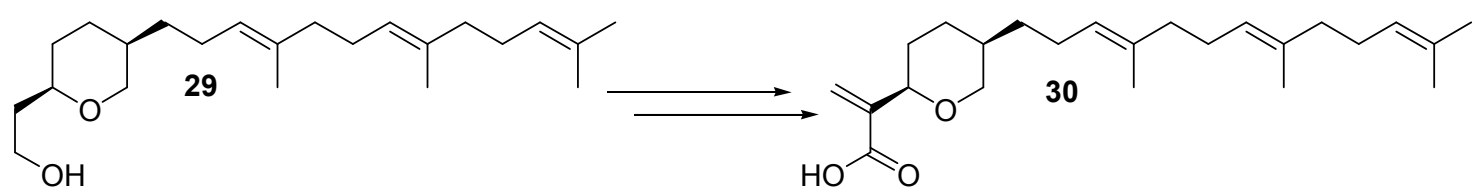

To a solution of oxalyl chloride $(60 \mu \mathrm{L}, 0.68 \mathrm{mmol})$ in anhydrous DCM (970 $\mu \mathrm{L})$ was added dropwise at $-78{ }^{\circ} \mathrm{C}$ a solution of DMSO $(100 \mu \mathrm{L}, 1.36 \mathrm{mmol})$ in anhydrous DCM $(970 \mu \mathrm{L})$. The reaction was stirred at $-78{ }^{\circ} \mathrm{C}$ for $20 \mathrm{~min}$. A solution of 29 (119 $\mathrm{mg}, 0.34 \mathrm{mmol})$ in anhydrous DCM $(850 \mu \mathrm{L})$ was added dropwise at $-78{ }^{\circ} \mathrm{C}$ over $15 \mathrm{~min}$ period and the reaction mixture was stirred for 1h at $-78{ }^{\circ} \mathrm{C} . \mathrm{Et}_{3} \mathrm{~N}(336 \mu \mathrm{L}, 2.39 \mathrm{mmol})$ was added dropwise and the reaction was stirred for another $1 \mathrm{~h}$ at $-78^{\circ} \mathrm{C}$. The cold bath was then removed and the reaction was stirred for $2 \mathrm{~h}$. Then reaction was quenched with brine, the aqueous layer was extracted two times with DCM. The combined organic extracts were washed with brine, dried over $\mathrm{Na}_{2} \mathrm{SO}_{4}$, filter on silica gel pad, and concentrated in vacuo to give the corresponding aldehyde as yellow oil that was characterized by ${ }^{1} \mathrm{H}$ NMR only (100 mg, 85\% crude). ${ }^{1} \mathrm{H}$ NMR (250 MHz, $\mathrm{CDCl}_{3}$ ): $\delta 9.78$ (dd, $\left.J=3.0 \mathrm{~Hz}, J=2.0 \mathrm{~Hz}, 1 \mathrm{H}, \mathrm{CH}_{2} \mathrm{CHO}\right), 5.15-5.04(\mathrm{~m}, 3 \mathrm{H}$, alkene- $H$ ), 3.93-3.81 (m, $1 \mathrm{H}$, pyran-OCH), $3.74(\mathrm{~d}, \mathrm{br}, \mathrm{J}=11.5 \mathrm{~Hz}, 1 \mathrm{H}$, pyran- 
$\mathrm{OCH}_{2}$ ), $3.58\left(\mathrm{dd}, J=11.5 \mathrm{~Hz}, J=11.5 \mathrm{~Hz}, 1 \mathrm{H}\right.$, pyran-OCH $\left.\mathrm{OCH}_{2}\right), 2.62$ (ddd, $J=$ $16.5 \mathrm{~Hz}, J=8.0 \mathrm{~Hz}, J=3.0 \mathrm{~Hz}, 1 \mathrm{H}, \mathrm{CH}_{2} \mathrm{CHO}$ ), 2.44 (ddd, $J=16.5 \mathrm{~Hz}, J=5.0$ $\left.\mathrm{Hz}, J=2.0 \mathrm{~Hz}, 1 \mathrm{H}, \mathrm{CH}_{2} \mathrm{CHO}\right), 2.13-1.93\left(\mathrm{~m}, 10 \mathrm{H}, \mathrm{CH}_{2}\right), 1.76-1.65(\mathrm{~m}, 3 \mathrm{H}$, $\left.\mathrm{CH}_{2}\right), 1.67\left(\mathrm{~s}, 3 \mathrm{H}\right.$, alkene- $\left.\mathrm{CH}_{3}\right), 1.64-1.57(\mathrm{~m}, 1 \mathrm{H}$, pyran- $\mathrm{CH}), 1.60(\mathrm{~s}, 9 \mathrm{H}$, alkene- $\left.\mathrm{CH}_{3}\right), 1.56-1.40\left(\mathrm{~m}, 3 \mathrm{H}, \mathrm{CH}_{2}\right)$.

The crude residue was dissolved in DCM $(7.0 \mathrm{~mL})$ and Eschenmoser's salt (266 mg, $1.44 \mathrm{mmol}), \mathrm{Et}_{3} \mathrm{~N}(82 \mu \mathrm{L}, 0.57 \mathrm{mmol})$ were added. The reaction was stirred at RT for $24 \mathrm{~h}$. The reaction was quenched with saturated aqueous $\mathrm{NaHCO}_{3}$ solution and the aqueous layer was extracted three times with DCM. The combined organic extracts were washed with brine, dried over $\mathrm{Na}_{2} \mathrm{SO}_{4}$ and concentrated in vacuo. After removal of DCM in vacuo, pentane was added in order to precipitate remaining Eschenmoser's salt. After filtration, the $\alpha-\beta$ unsaturated aldehyde was concentrated in vacuo and was obtained as a yellow oil (103 mg, 100\% crude).

The crude residue was dissolved in $\mathrm{t}-\mathrm{BuOH} / \mathrm{H}_{2} \mathrm{O}(4: 1,11.6 \mathrm{~mL}: 3 \mathrm{~mL})$. To the solution was added at RT 2-methyl-2-butene (2M in THF, $36 \mathrm{~mL}, 71.9 \mathrm{mmol}$ ), $\mathrm{NaH}_{2} \mathrm{PO}_{4}(120.5 \mathrm{mg}, 1.0 \mathrm{mmol})$, and $\mathrm{NaClO}_{2}(113 \mathrm{mg}, 1.00 \mathrm{mmol})$. The reaction was stirred at RT for 2-3 $\mathrm{h}$. After this period, brine was added and the reaction mixture extracted three times with EtOAc. The combined organic layer was dried over $\mathrm{Na}_{2} \mathrm{SO}_{4}$ and concentrated in vacuo. The crude residue was purified by flash chromatography (gradient starting with 90:10 and ending with 60:40 petroleum ether/ $\left.\mathrm{Et}_{2} \mathrm{O}\right)$, to give the desired product 30 as a yellow oil $(51 \mathrm{mg}$, $40 \%$ over three steps). The compound showed satisfactory spectra data ${ }^{\mathrm{xi}}:{ }^{1} \mathrm{H}$ NMR (250 MHz, CDCl 3 ): $\delta 6.39$ (s, br, 1H, $\mathrm{HO}_{2}$ C-alkene-H), 5.95 (t, J = $1.0 \mathrm{~Hz}$, $1 \mathrm{H}, \mathrm{HO}_{2}$ C-alkene- $H$ ), 5.17-5.04 (m, 3H, alkene- $H$ ), $4.21(\mathrm{~d}, J=10.5 \mathrm{~Hz}$, pyran$\mathrm{OCH}$ ), 3.90 (d, $J=11.5 \mathrm{~Hz}$, pyran-OCH ), 3.69 (dd, $J=11.5 \mathrm{~Hz}, J=2.0 \mathrm{~Hz}, 1 \mathrm{H}$, pyran-OCH $\left.\mathrm{O}_{2}\right), 2.14-1.91\left(\mathrm{~m}, 10 \mathrm{H}, \mathrm{CH}_{2}\right), 1.82-1.65\left(\mathrm{~m}, 3 \mathrm{H}, \mathrm{CH}_{2}\right), 1.68(\mathrm{~s}, 3 \mathrm{H}$, alkene- $\left.\mathrm{CH}_{3}\right), 1.64-1.40\left(\mathrm{~m}, 4 \mathrm{H}, \mathrm{CH}, \mathrm{CH}_{2}\right), 1.60\left(\mathrm{~s}, 9 \mathrm{H}\right.$, alkene- $\left.\mathrm{CH}_{3}\right) ;{ }^{13} \mathrm{C} \mathrm{NMR}$ $\left(62.9 \mathrm{MHz}, \mathrm{CDCl}_{3}\right): \delta 169.4,141.0,135.6,135.1,131.4,127.1,124.5,124.3$ (2C), 76.4, 71.9, 39.9 (2C), 32.7, 30.2, 27.3 (2C), 26.9, 26.7, 25.9, 25.8, 17.8, 16.2 (2C); IR (film, cm ${ }^{-1}$ ): 2926 (s), 2856 (s), 1694 (s), 1628 (m), 1441 (m), 
1378 (w), 1286 (w), 1168 (w), 1109 (m), 1065 (m), $1041(\mathrm{~m})$; HRMS (EI): m/z $[\mathrm{M}]^{+}$calcd for $\mathrm{C}_{24} \mathrm{H}_{38} \mathrm{O}_{3}: 374.2821$, found: $374.2811,[\alpha]_{D}{ }^{22}=+56.0$ (c $=0.25$ in $\mathrm{CHCl}_{3}$, lit. ${ }^{14}[\alpha]_{D}^{25}=+55.0\left(\mathrm{c}=0.23\right.$ in $\left.\mathrm{CHCl}_{3}\right)$. 

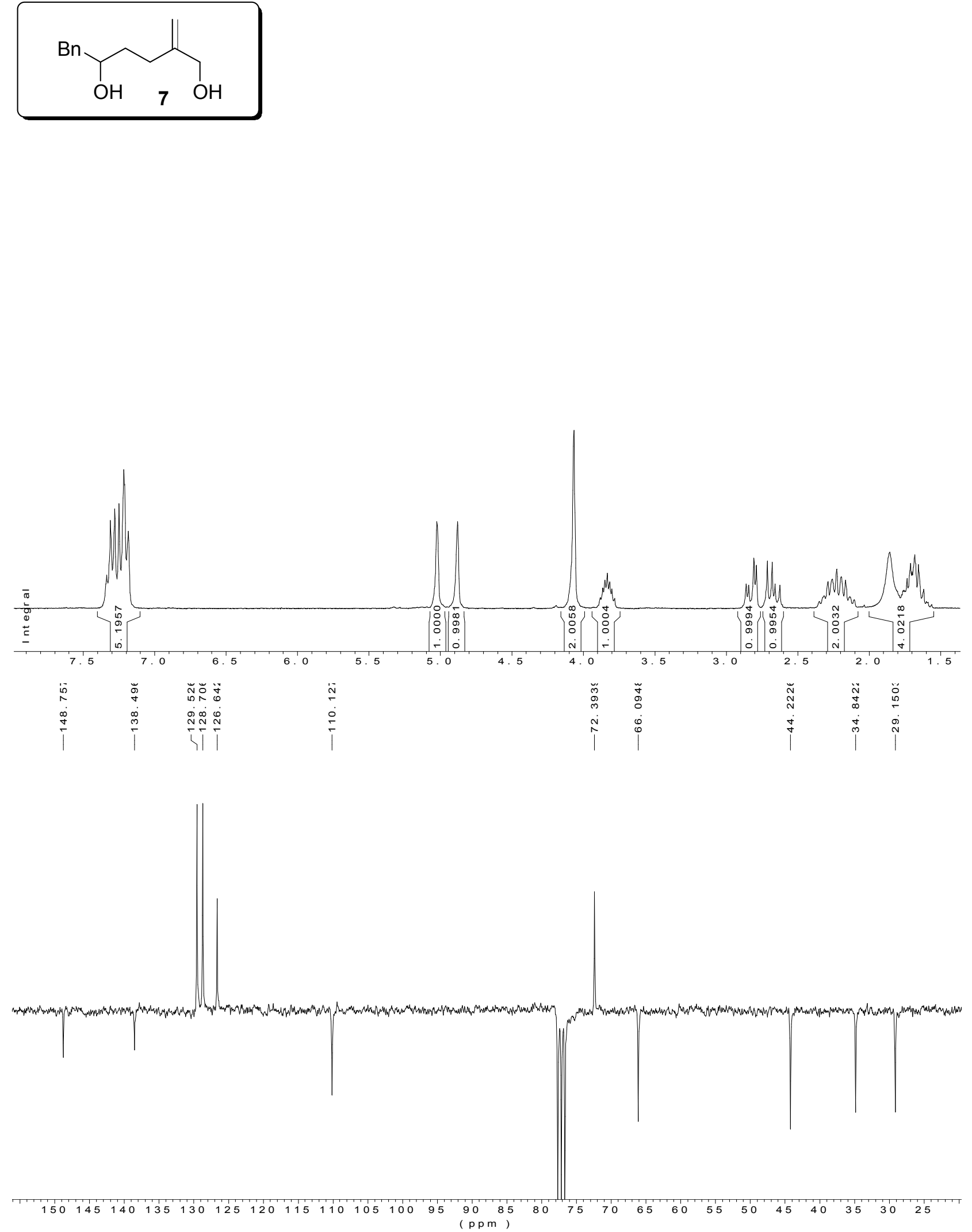

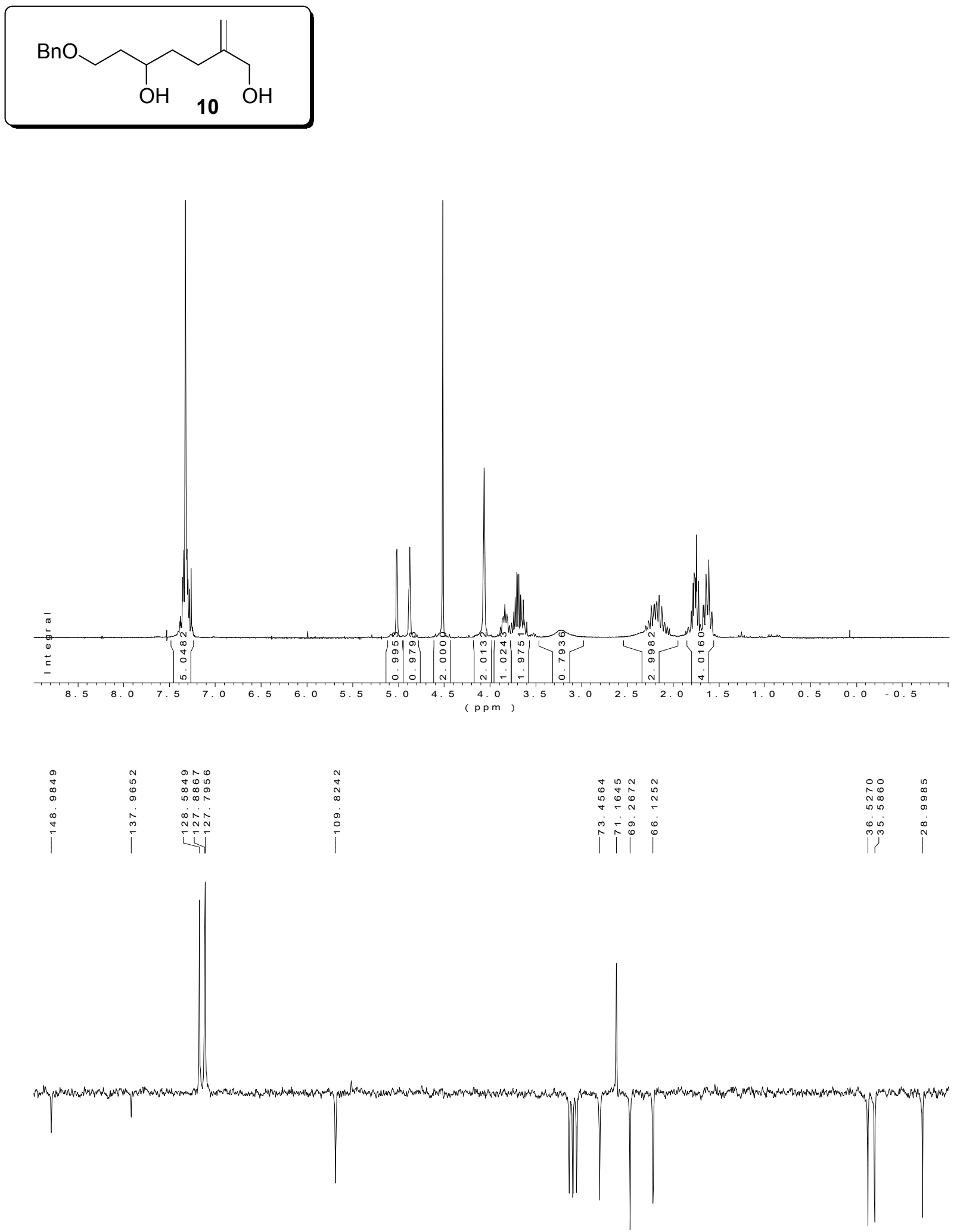

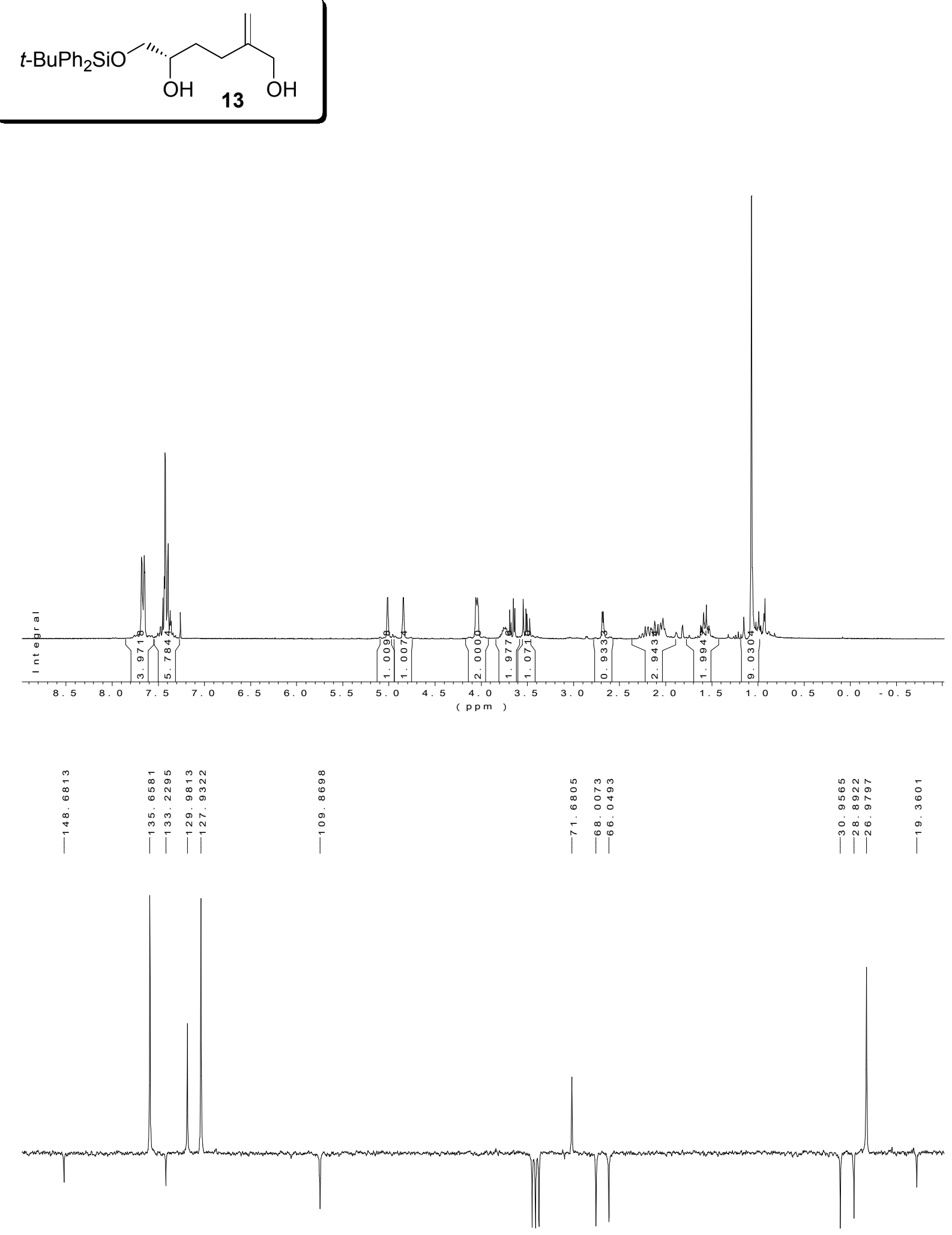

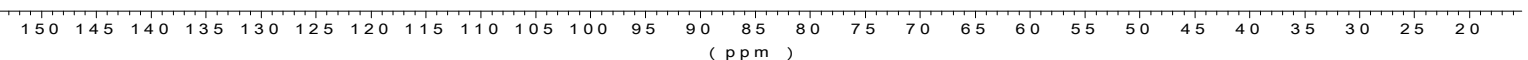



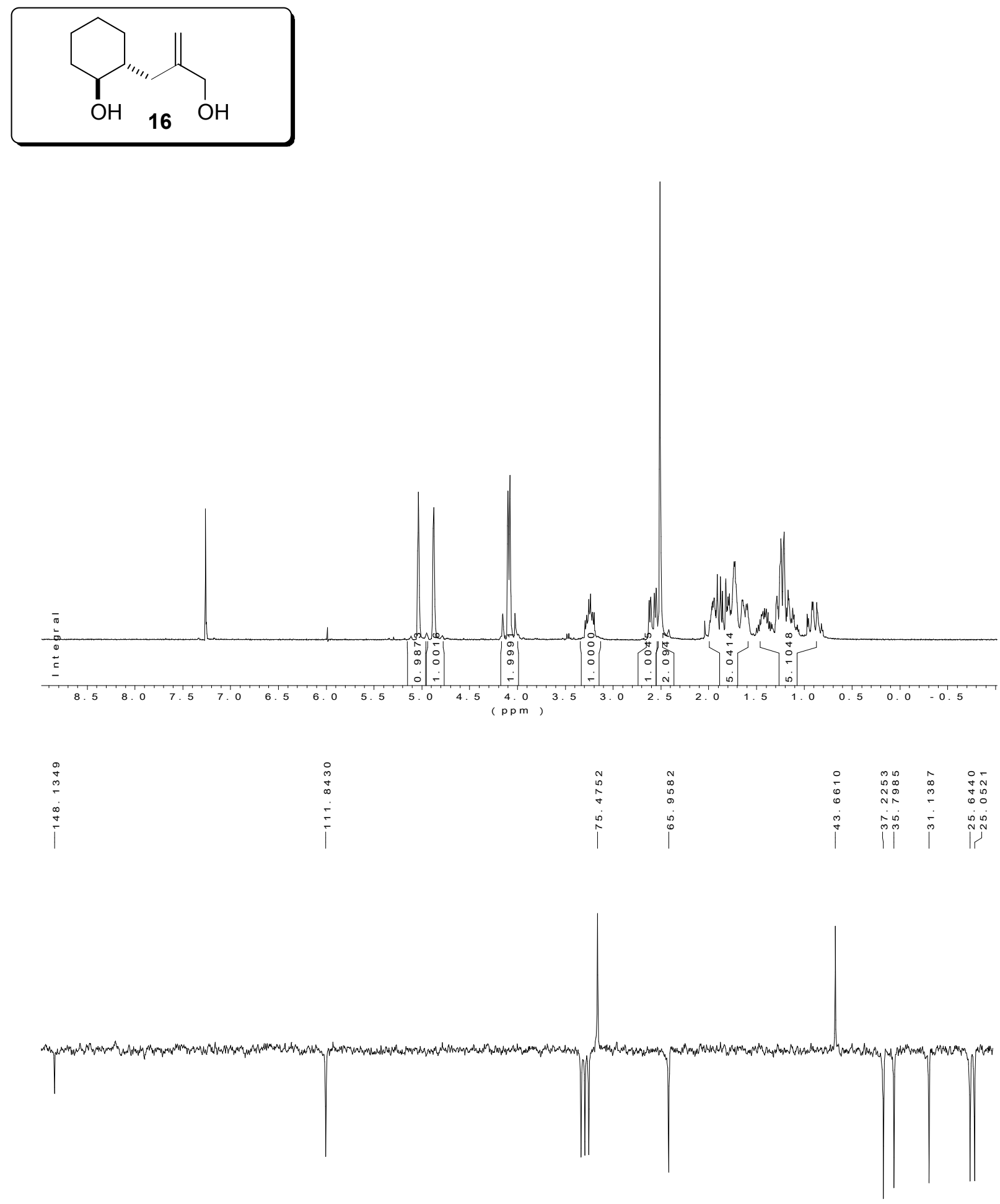

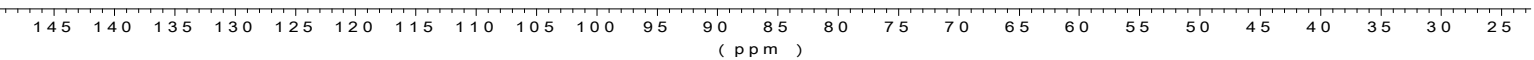



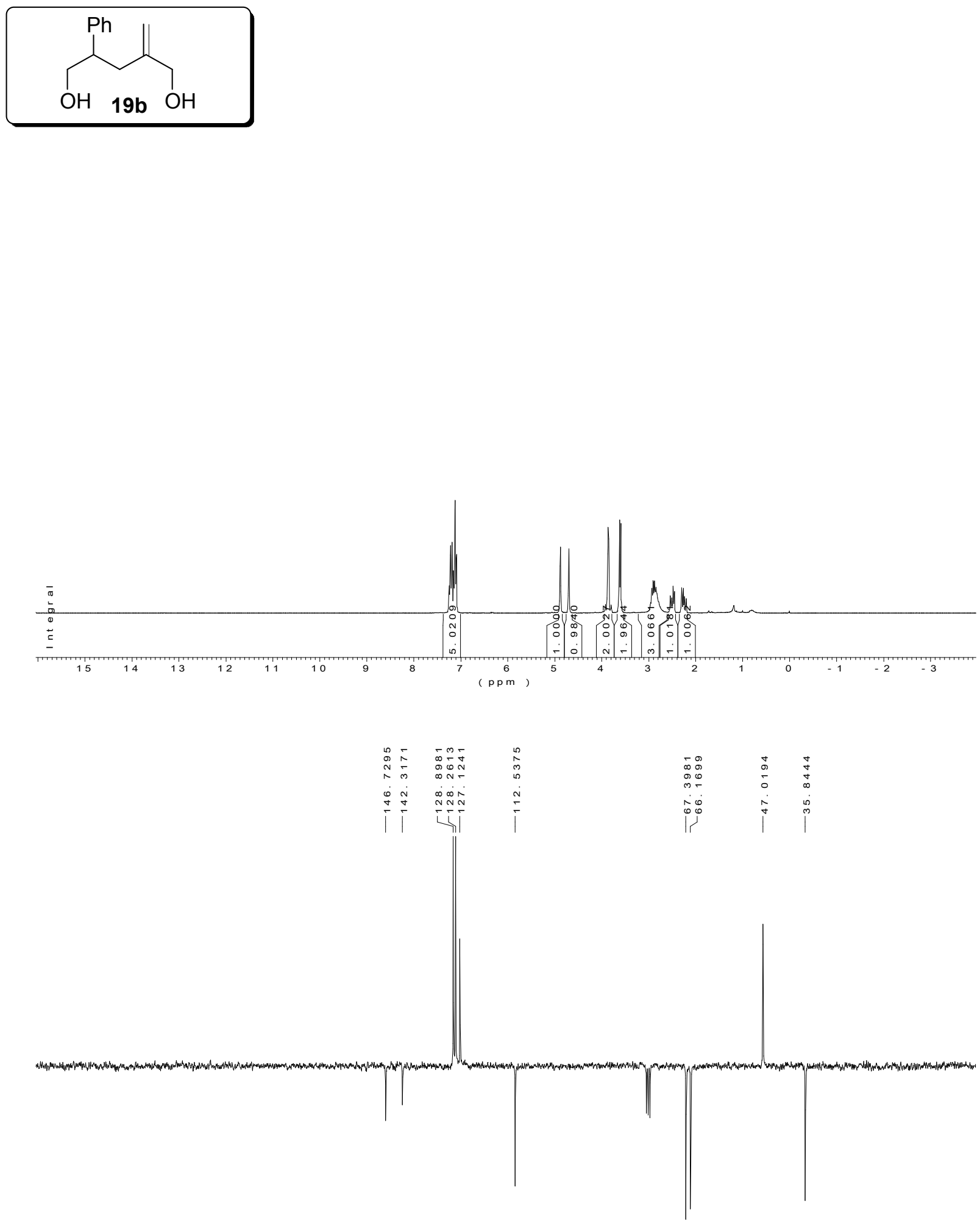

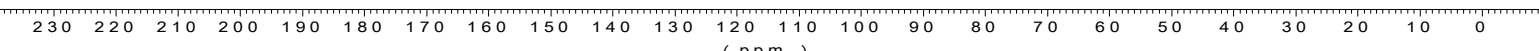
$(\mathrm{ppm})$ 

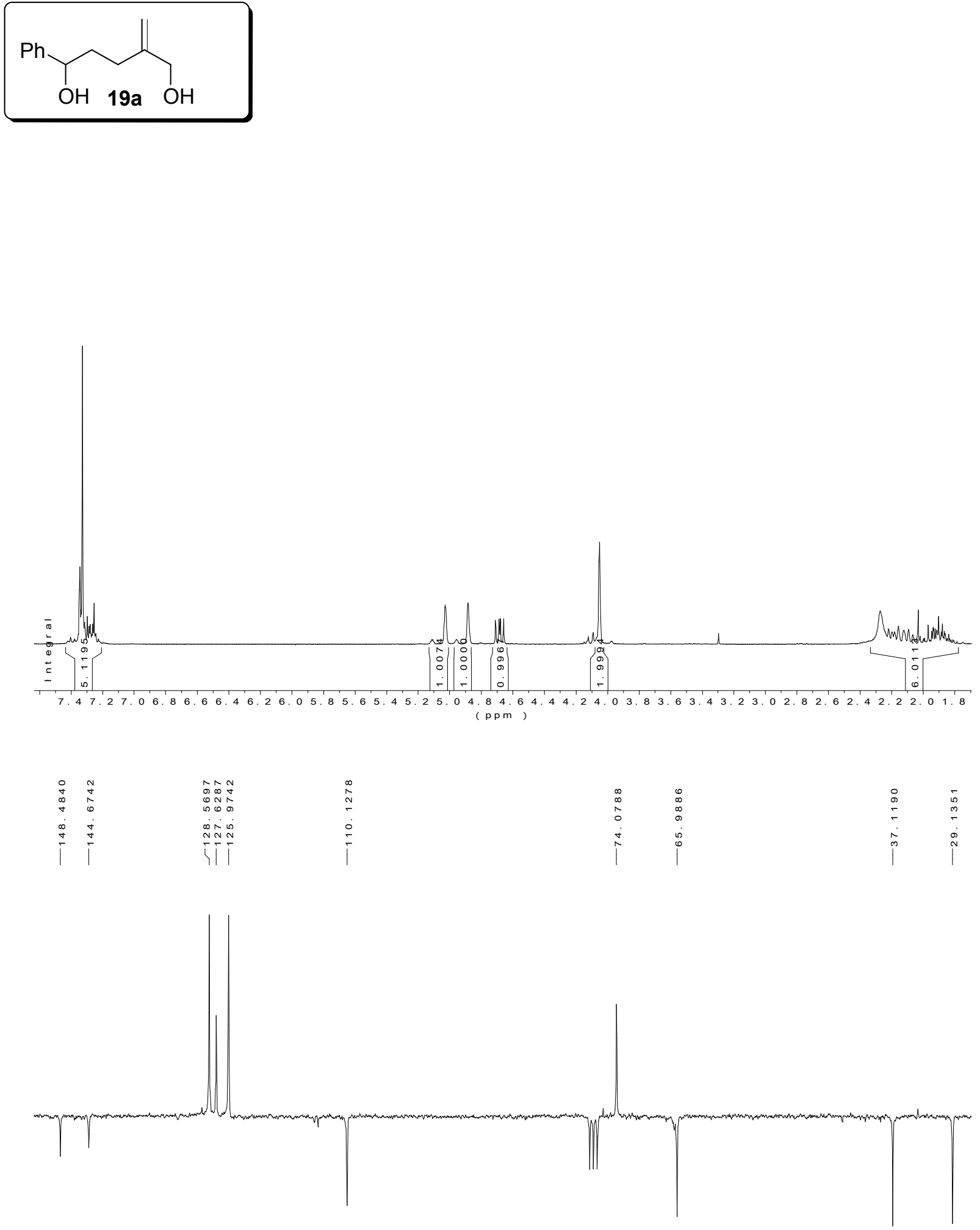

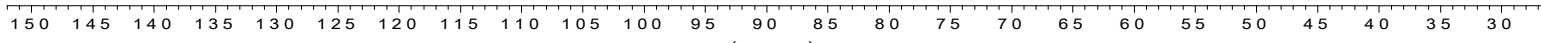
( $p p m)$ 

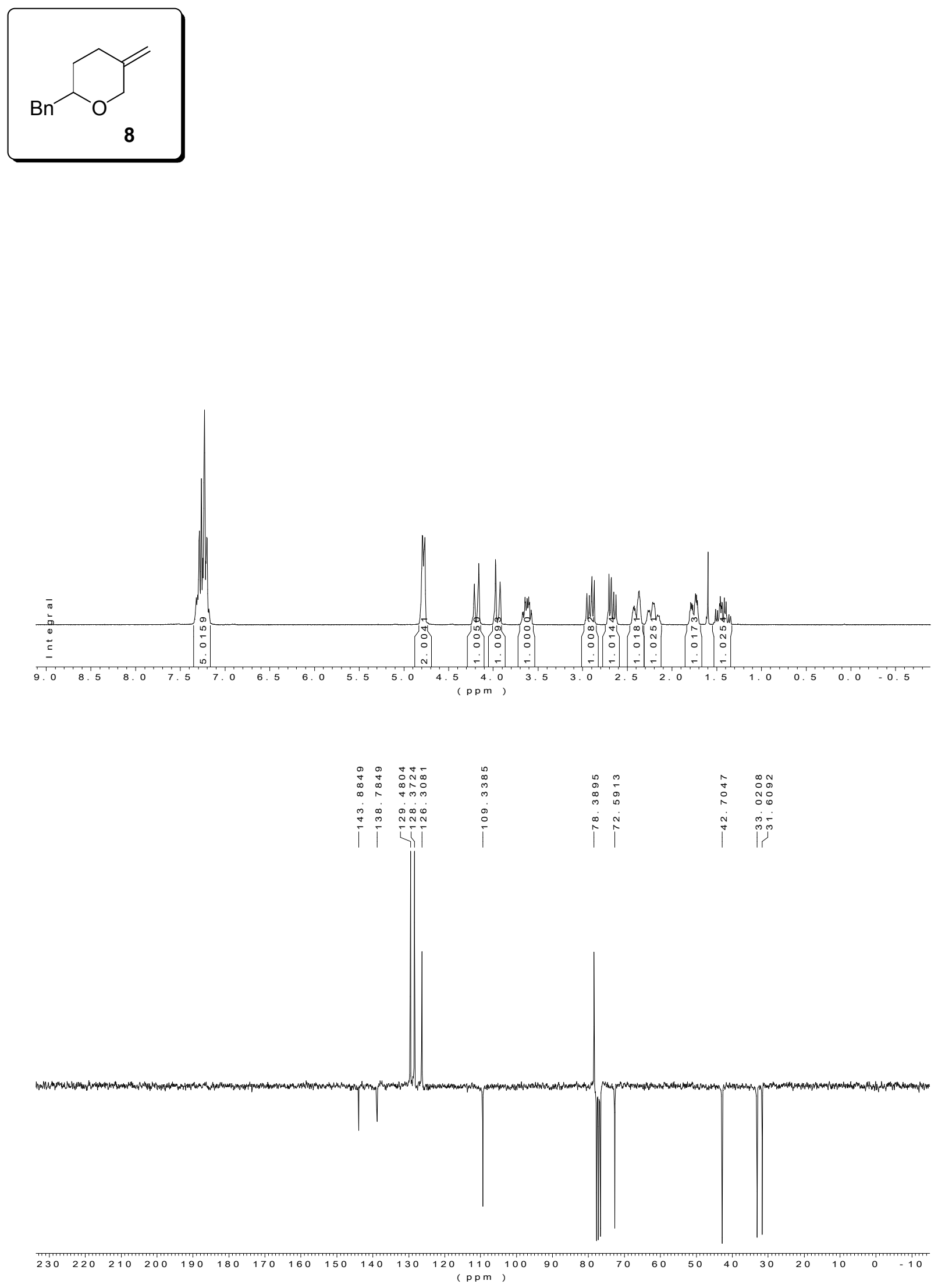

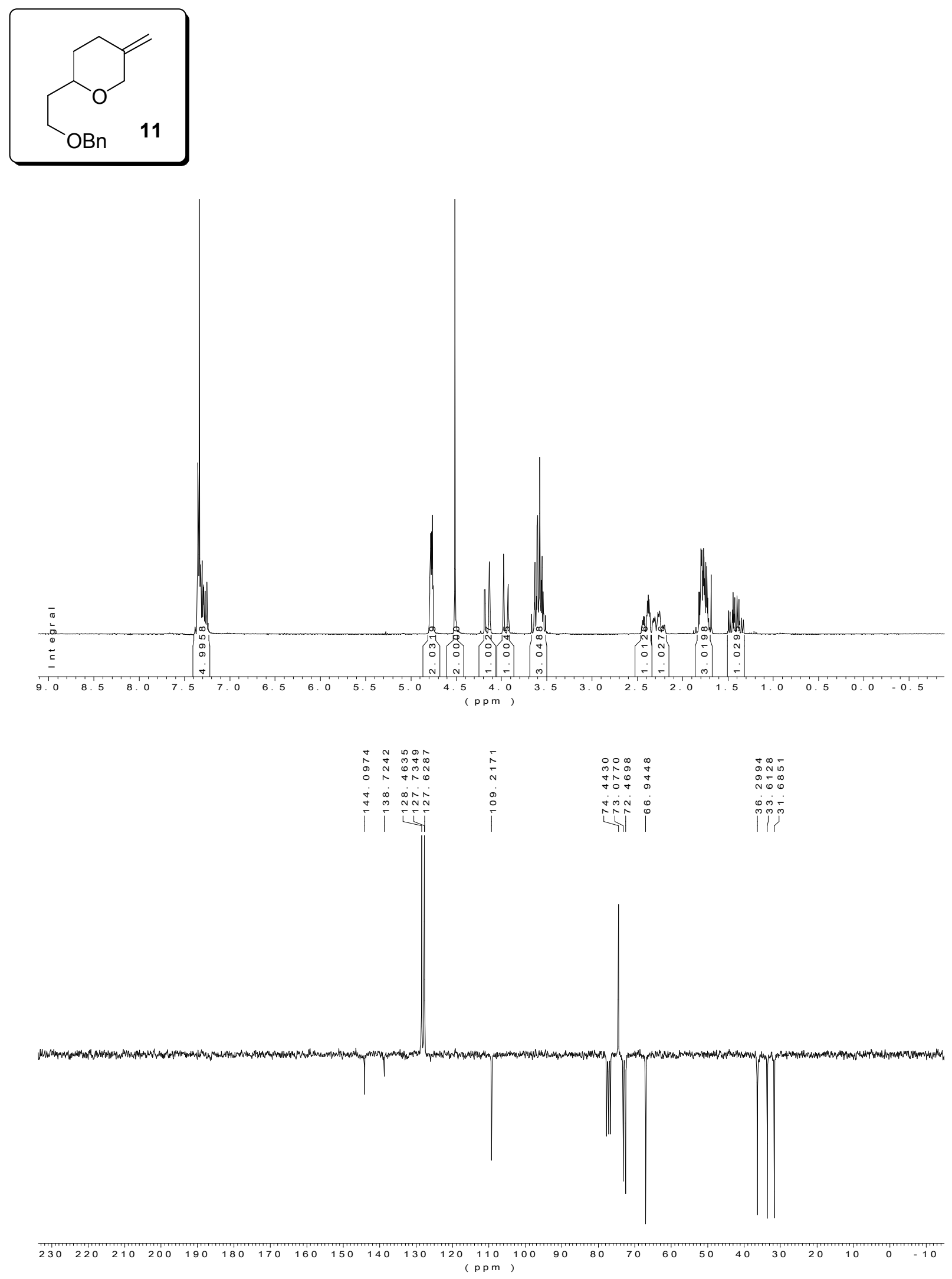

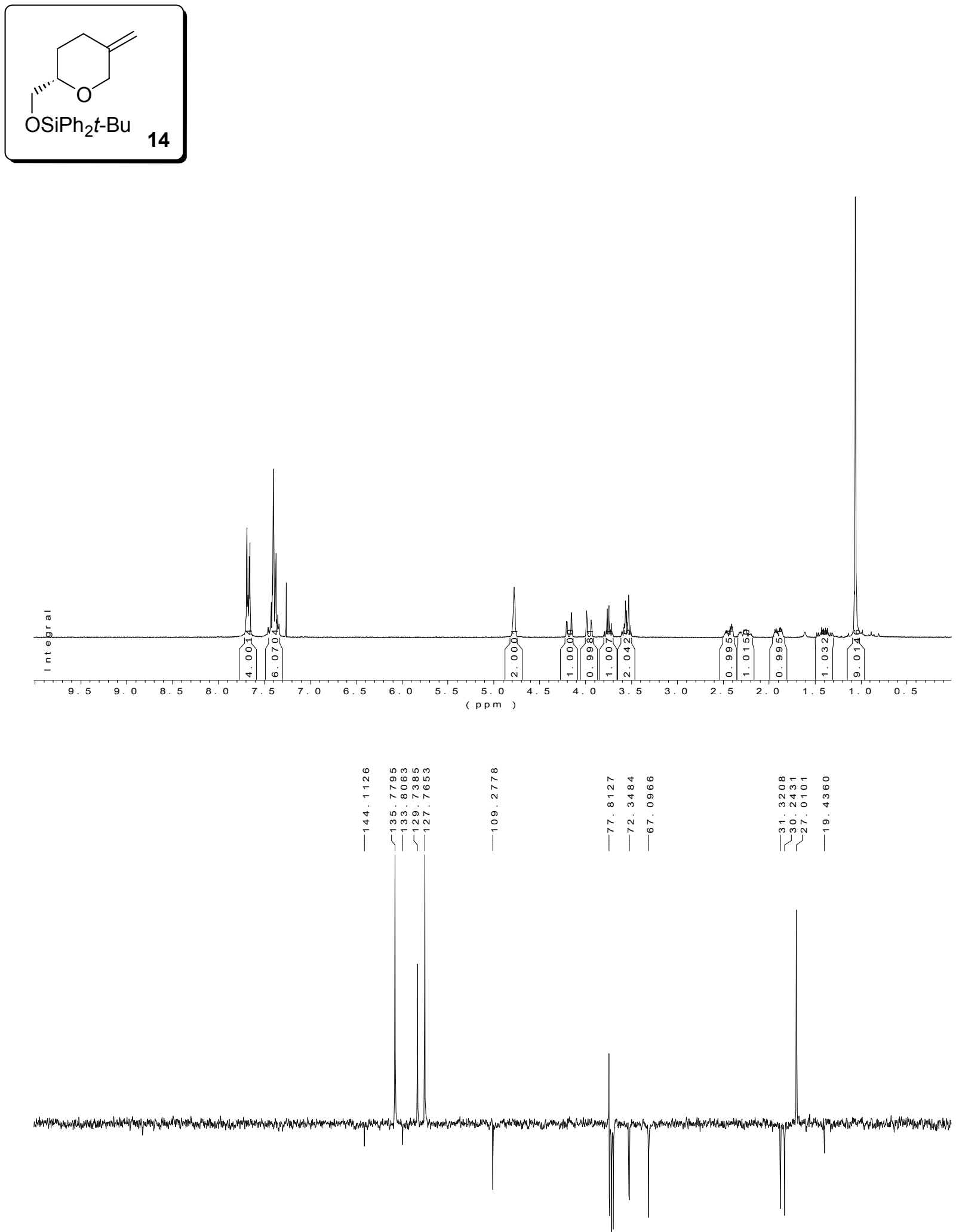

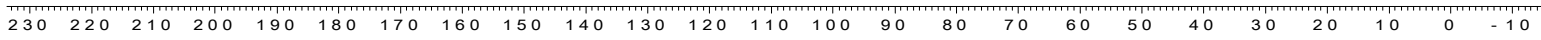
( $\mathrm{ppm}$ ) 

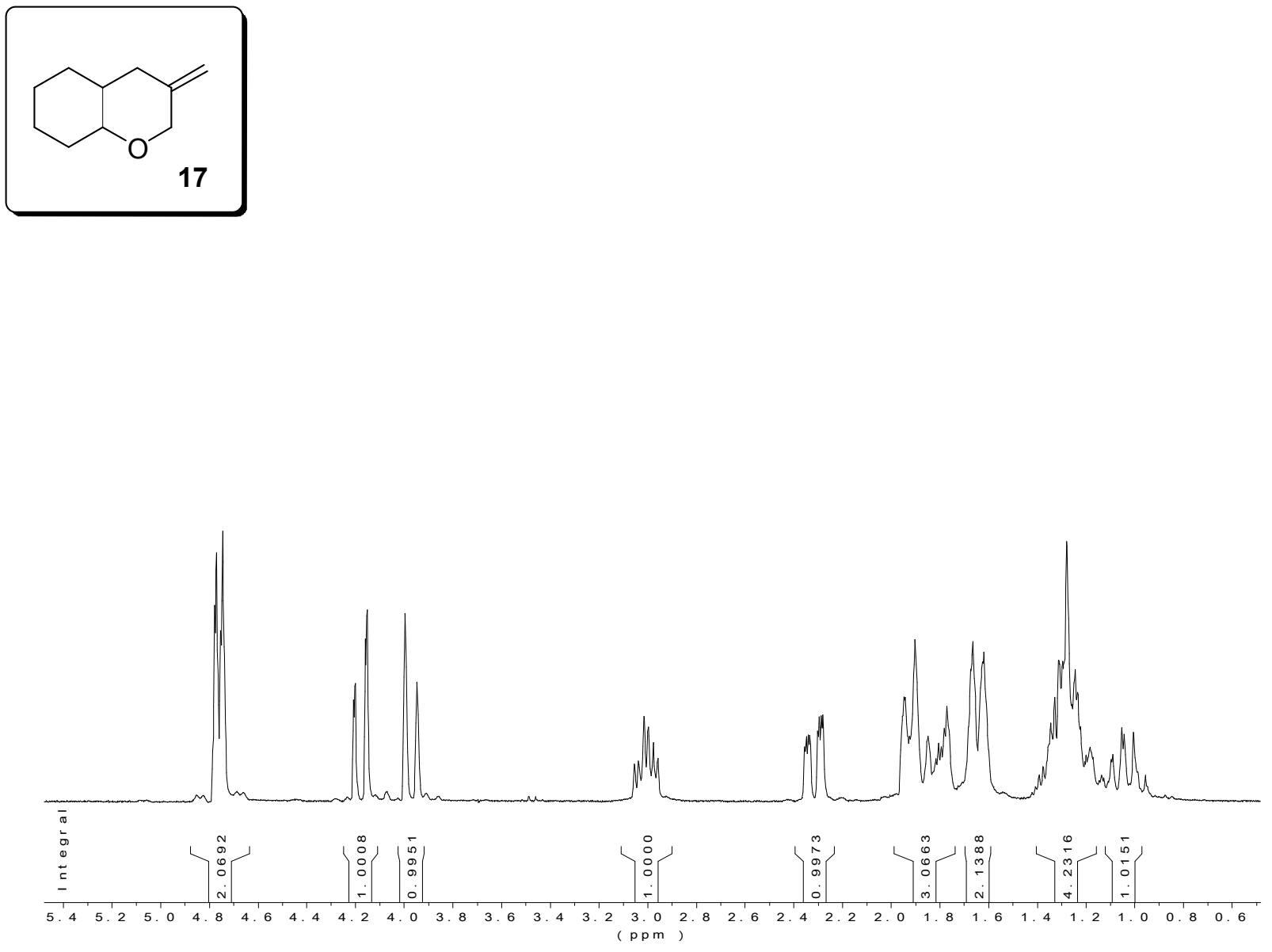

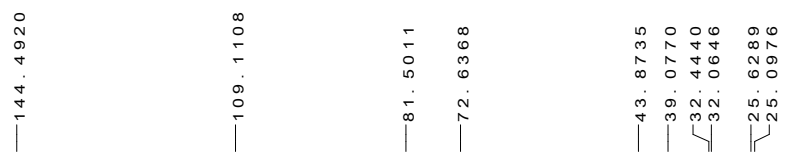

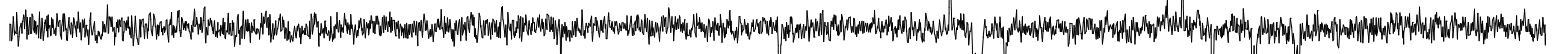

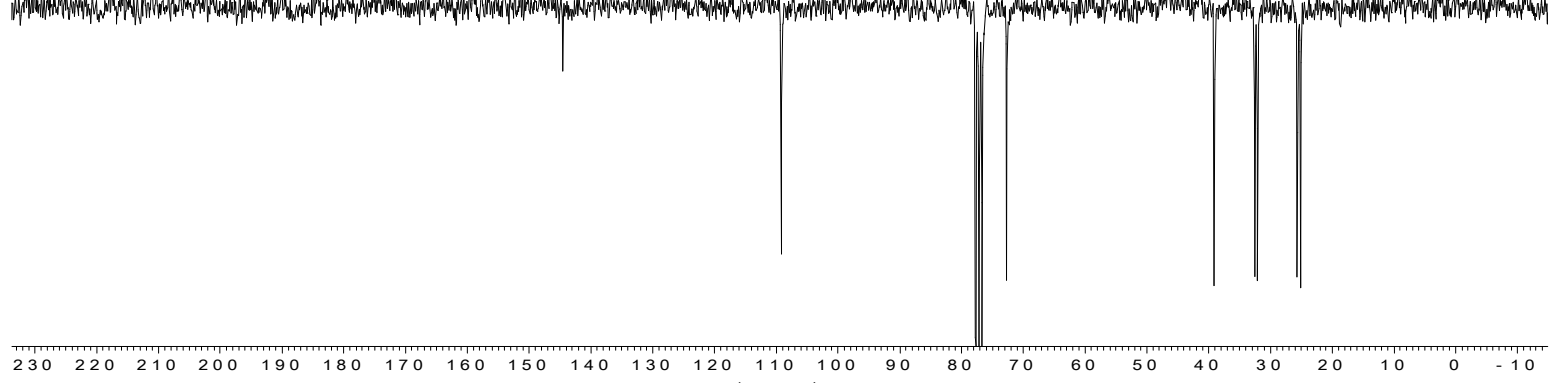



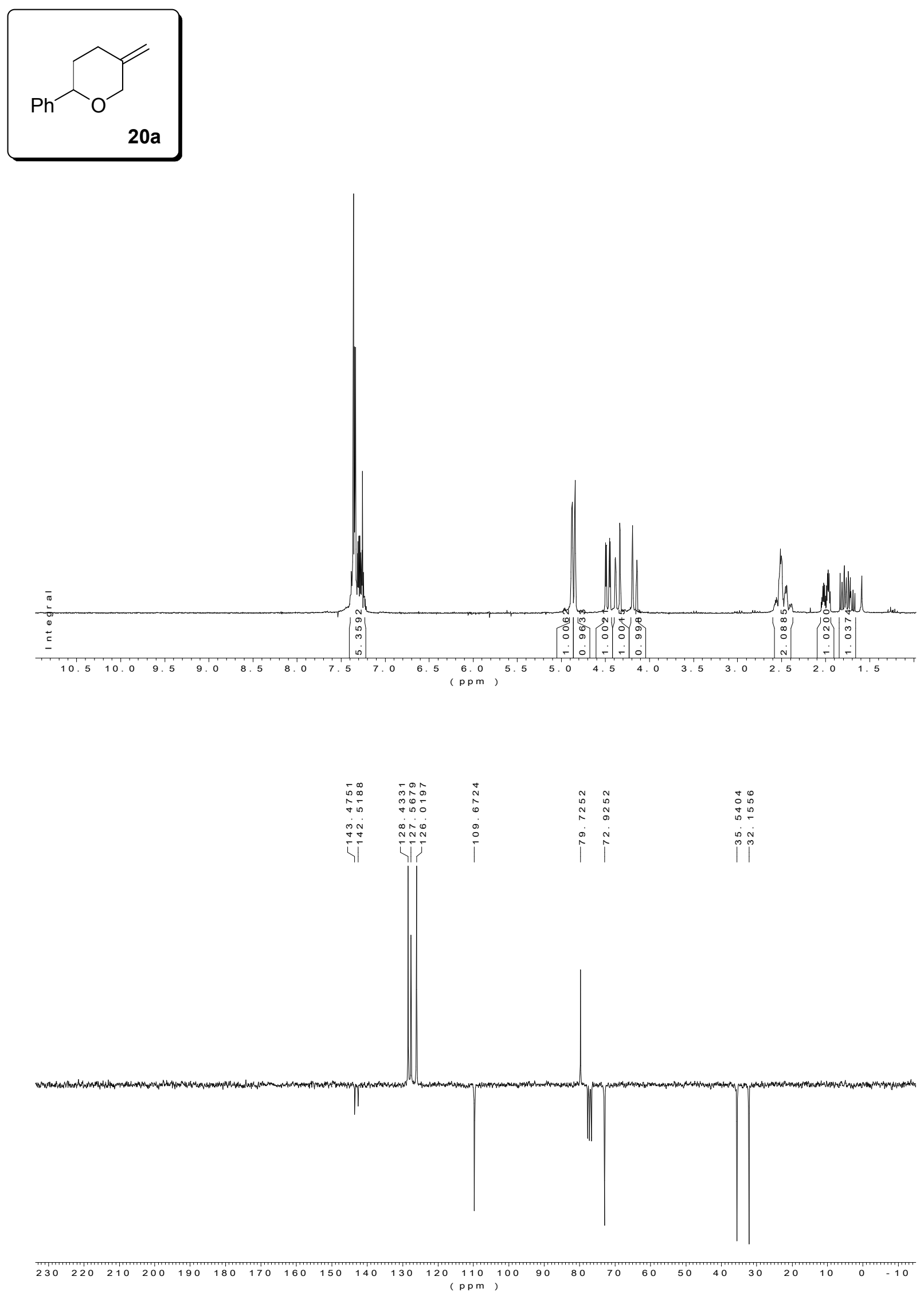

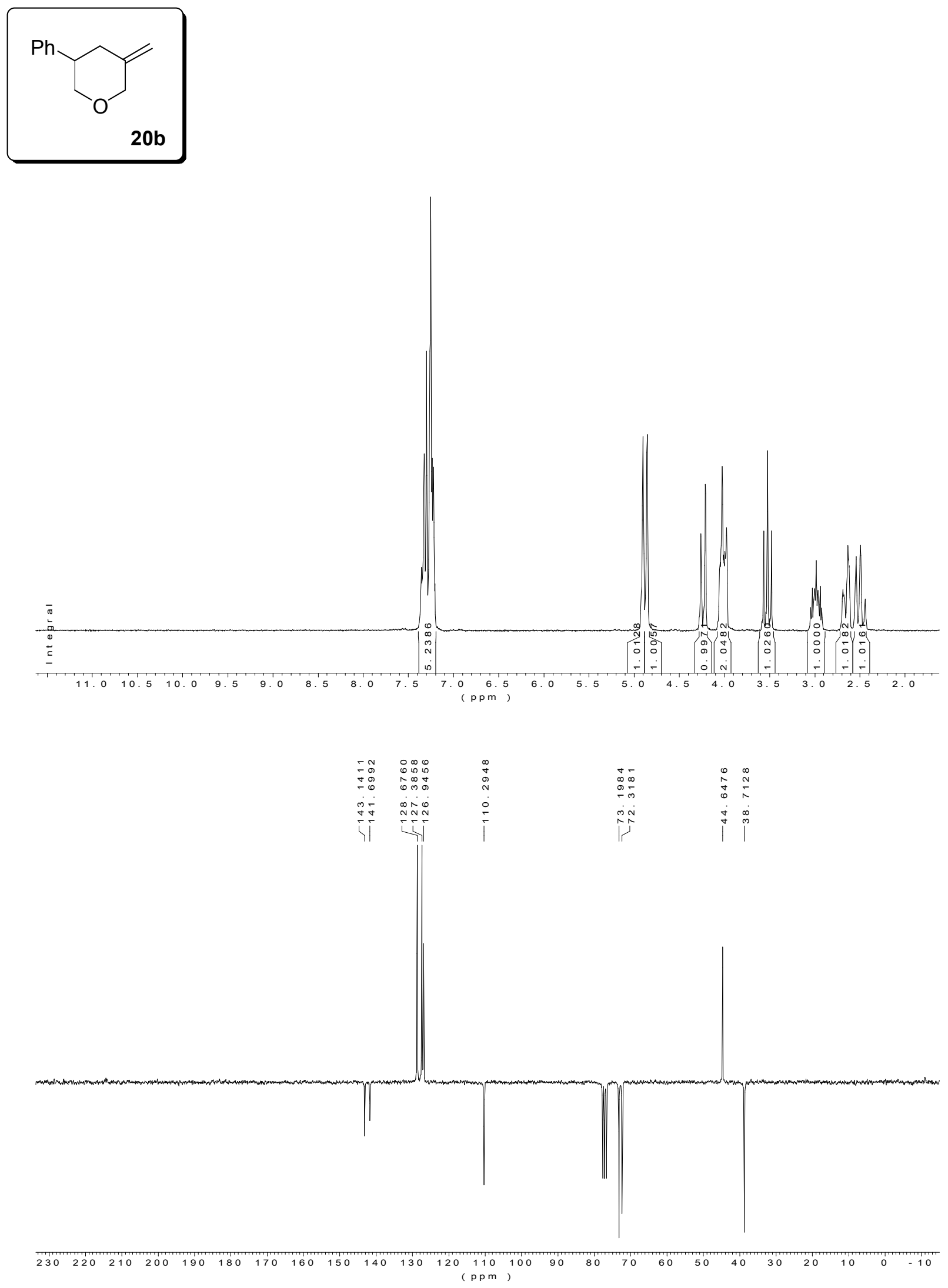

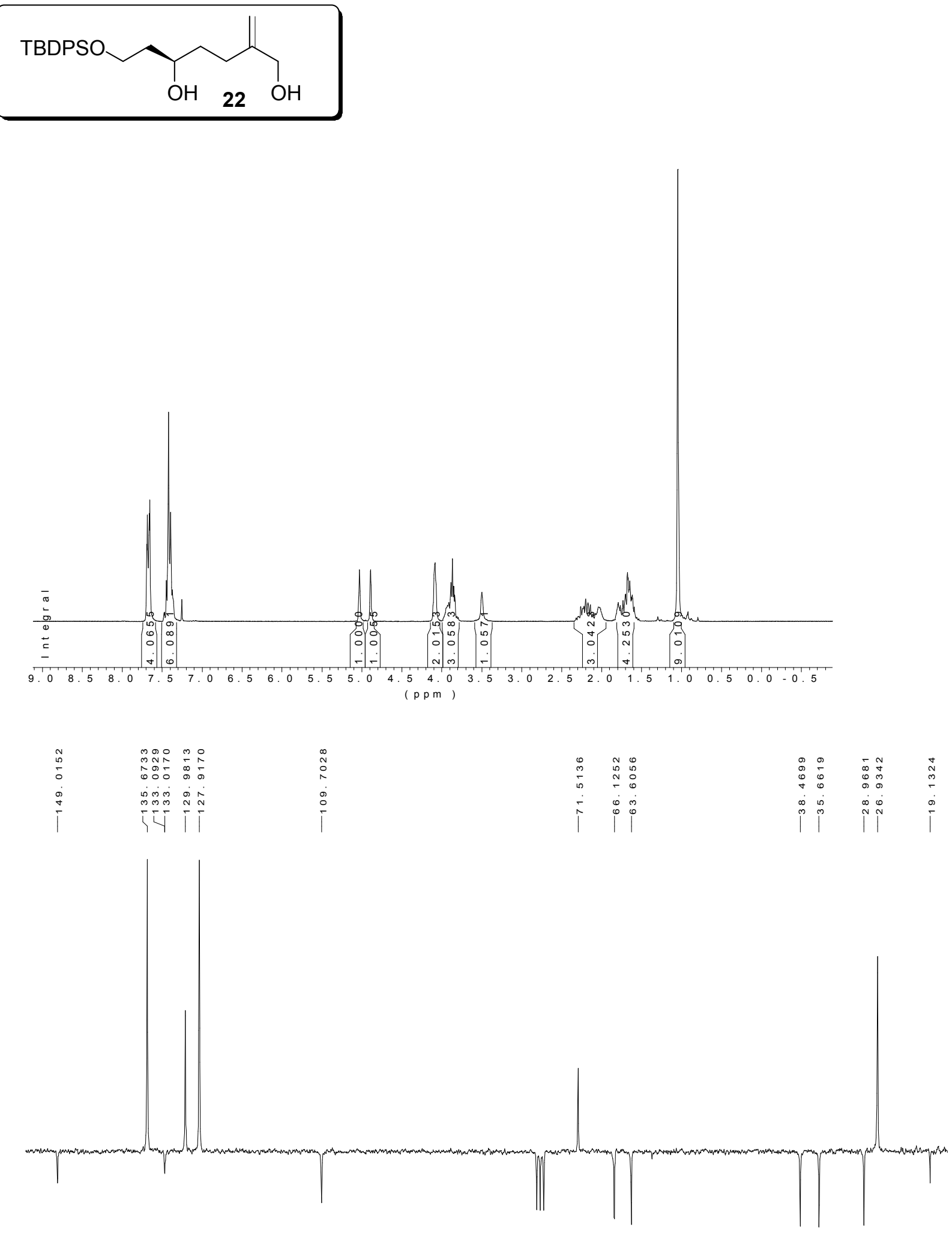

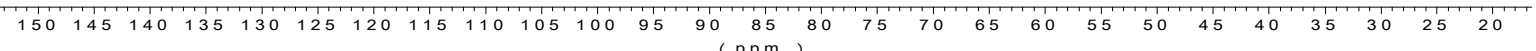



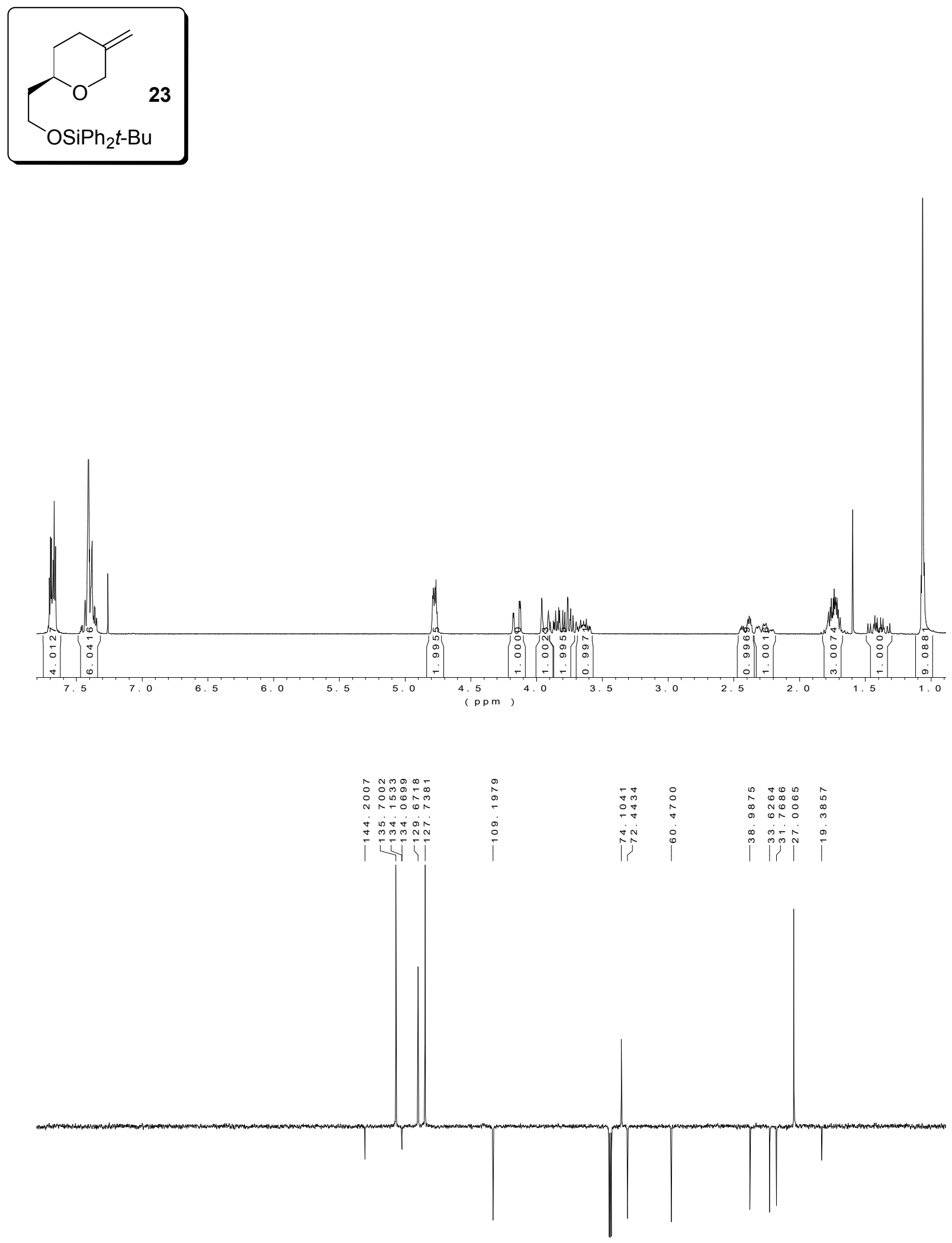

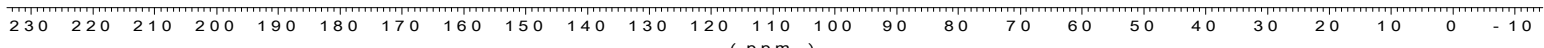
( ppm ) 


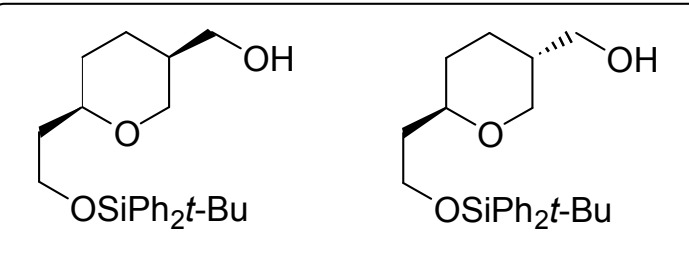

24; 3:1 mixture
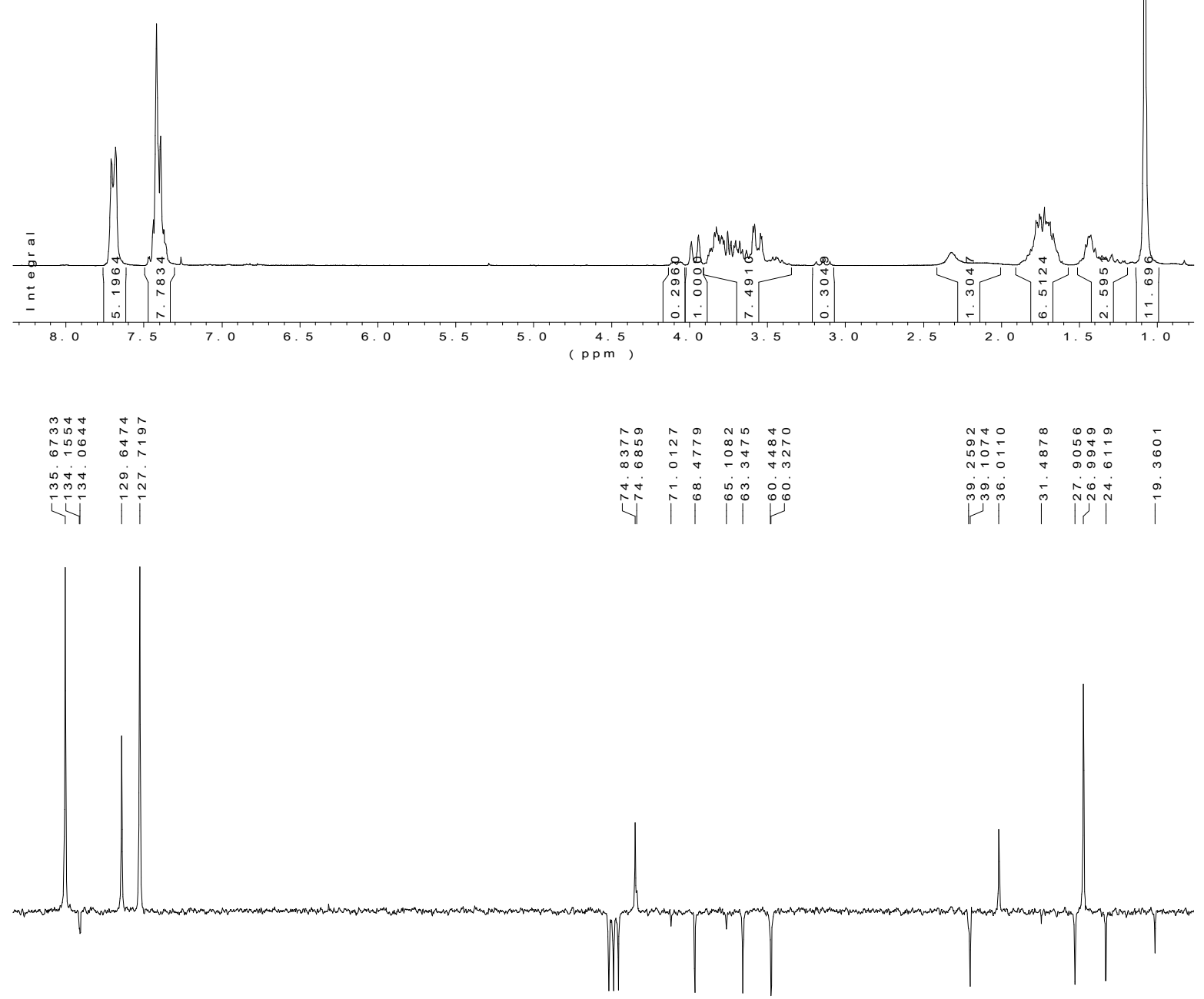

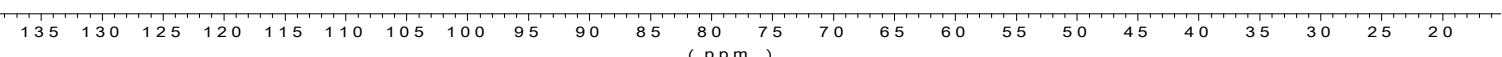



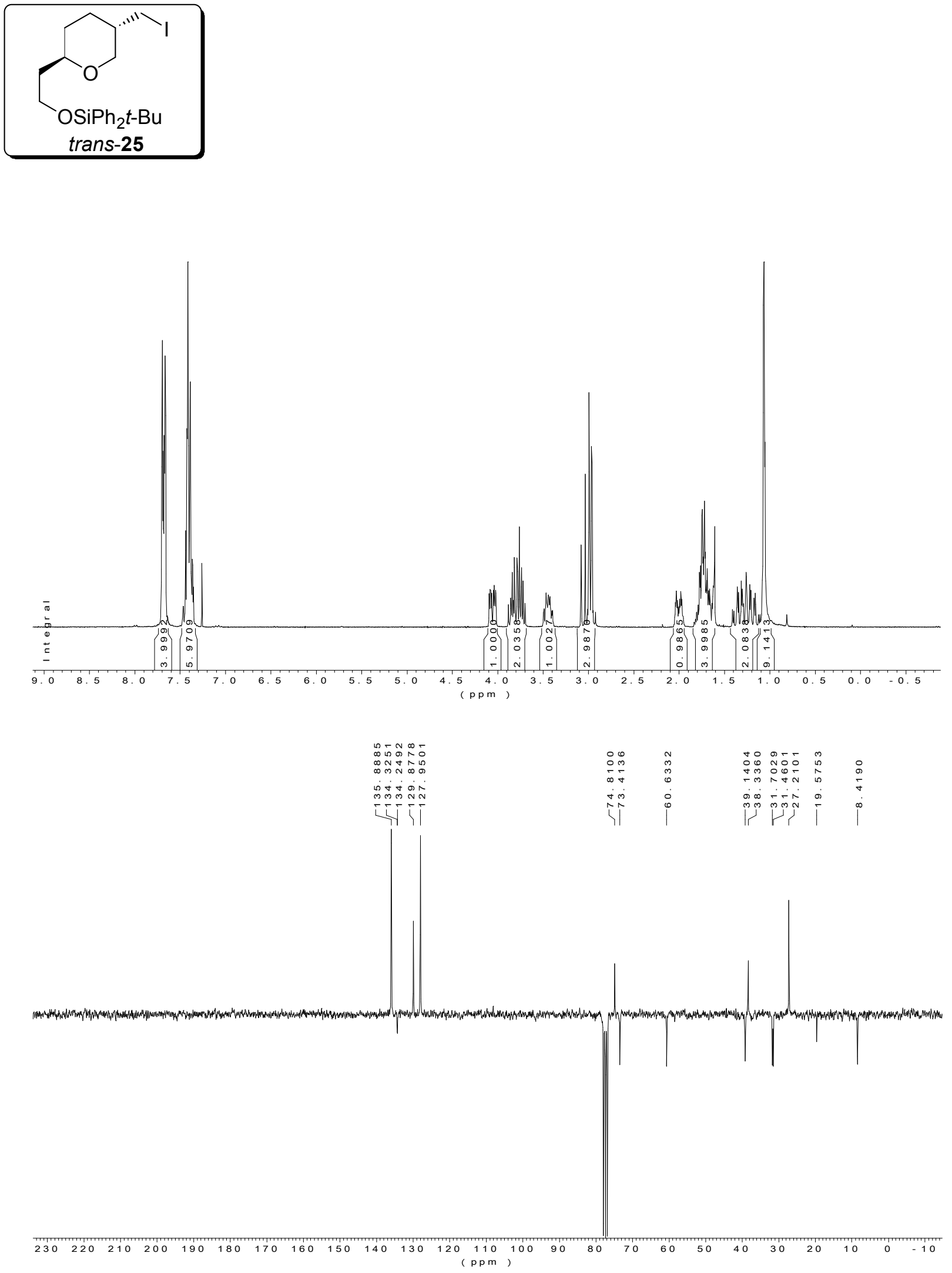

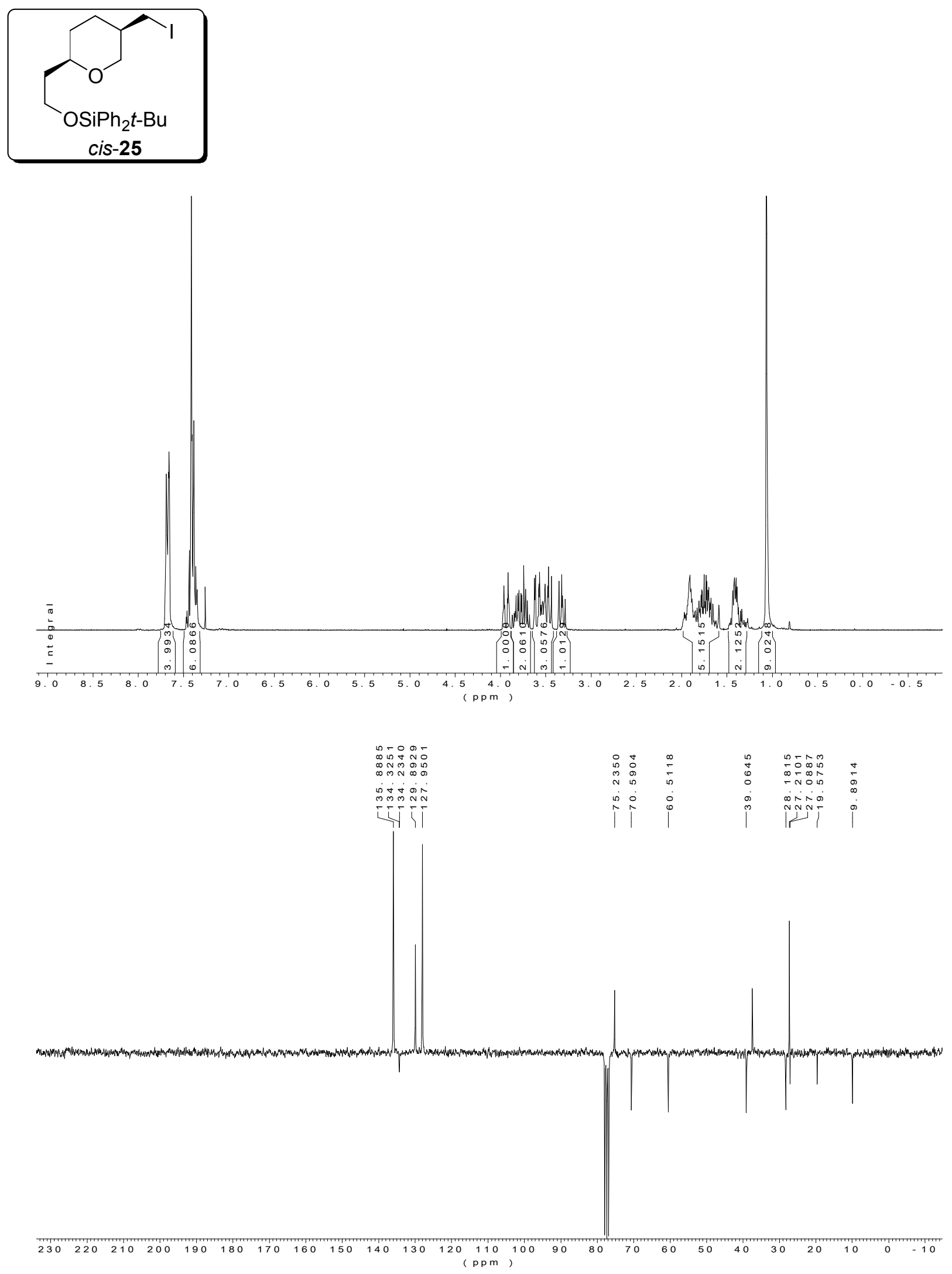

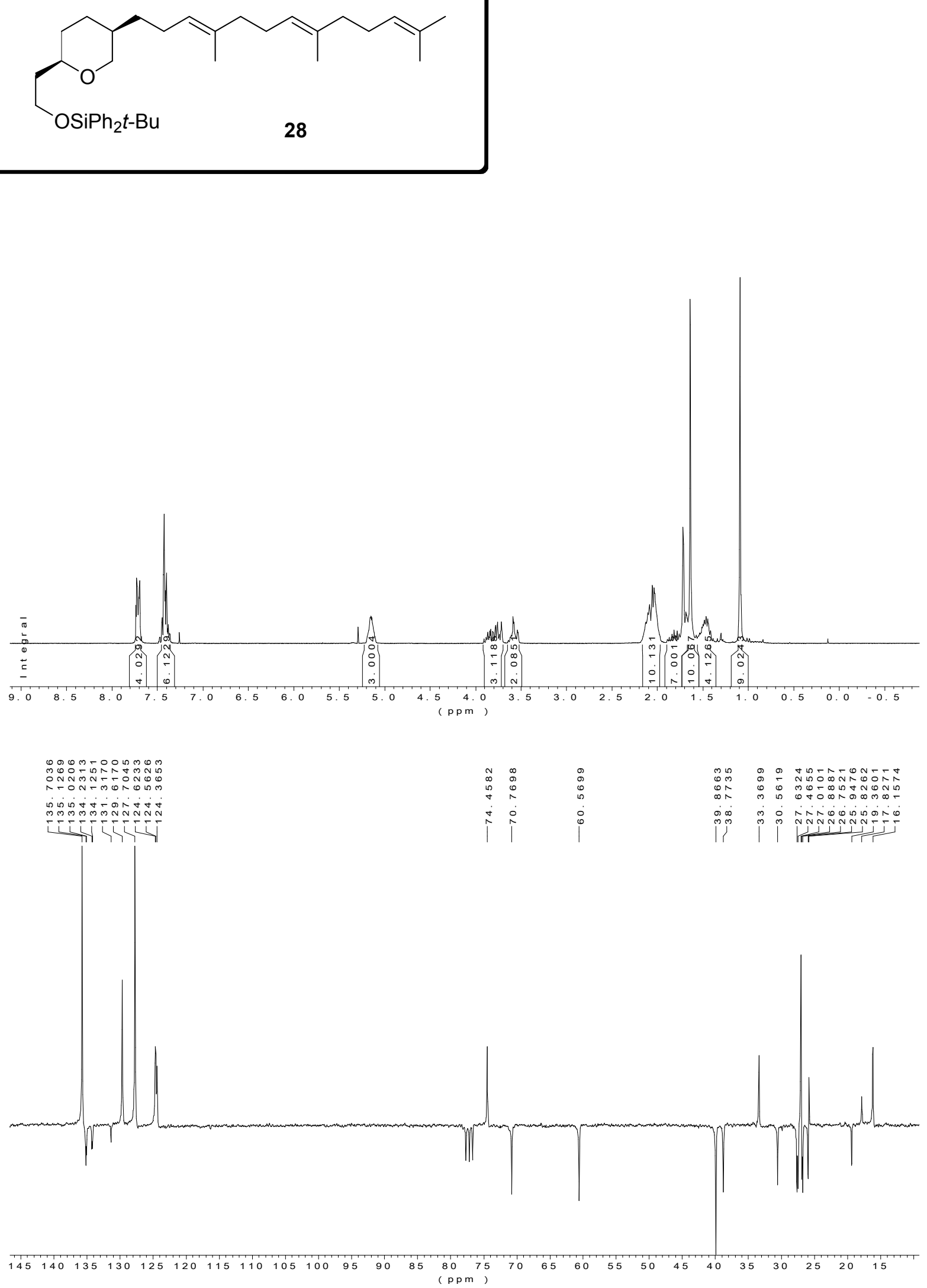


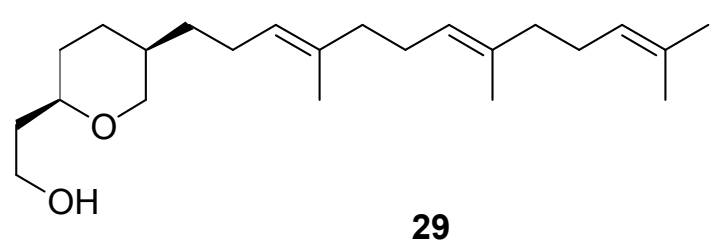

29
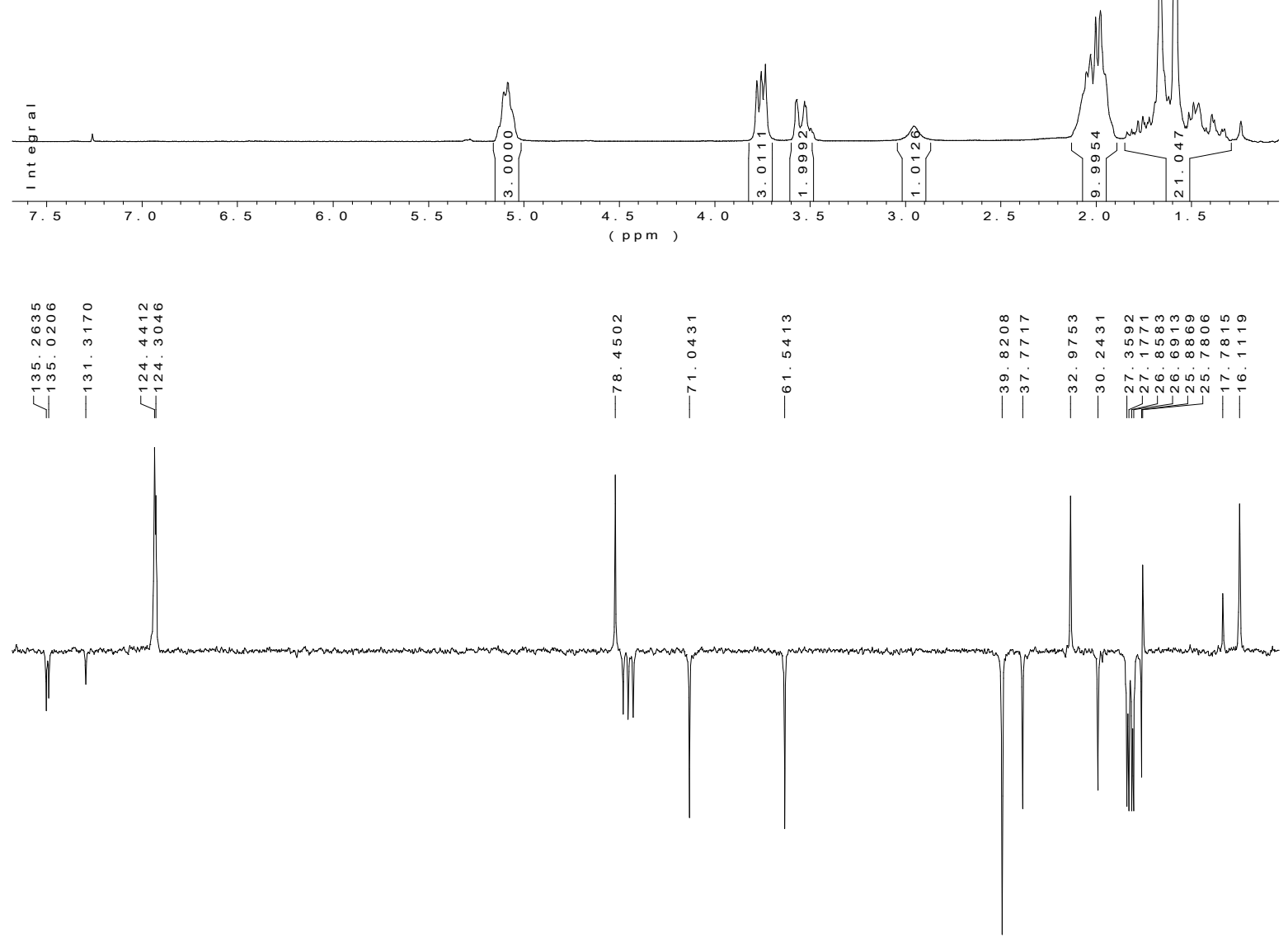

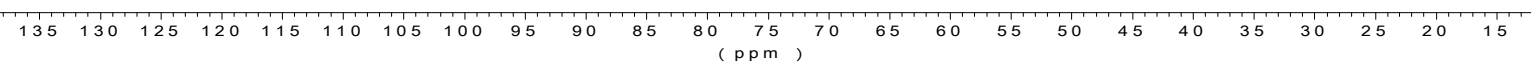



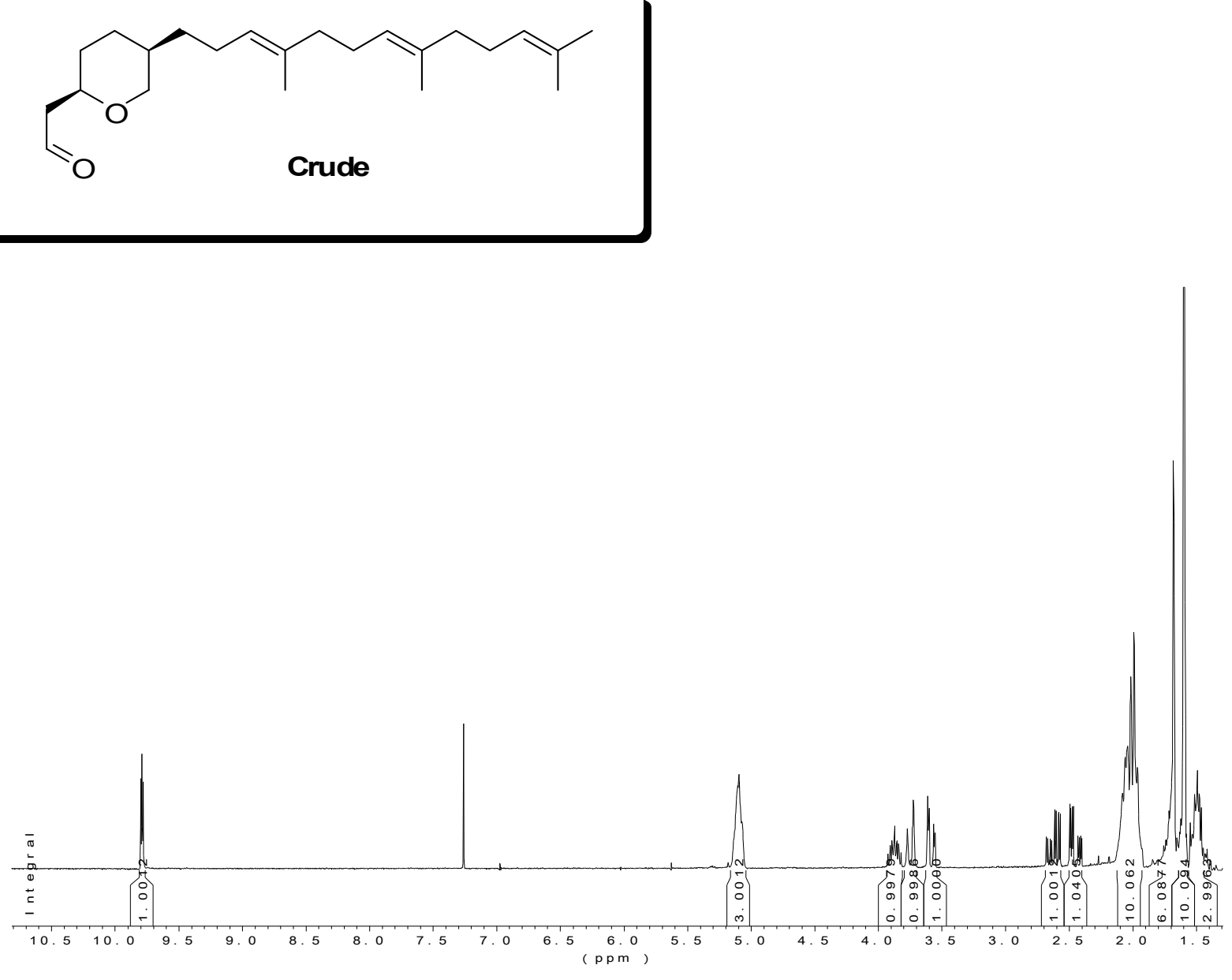

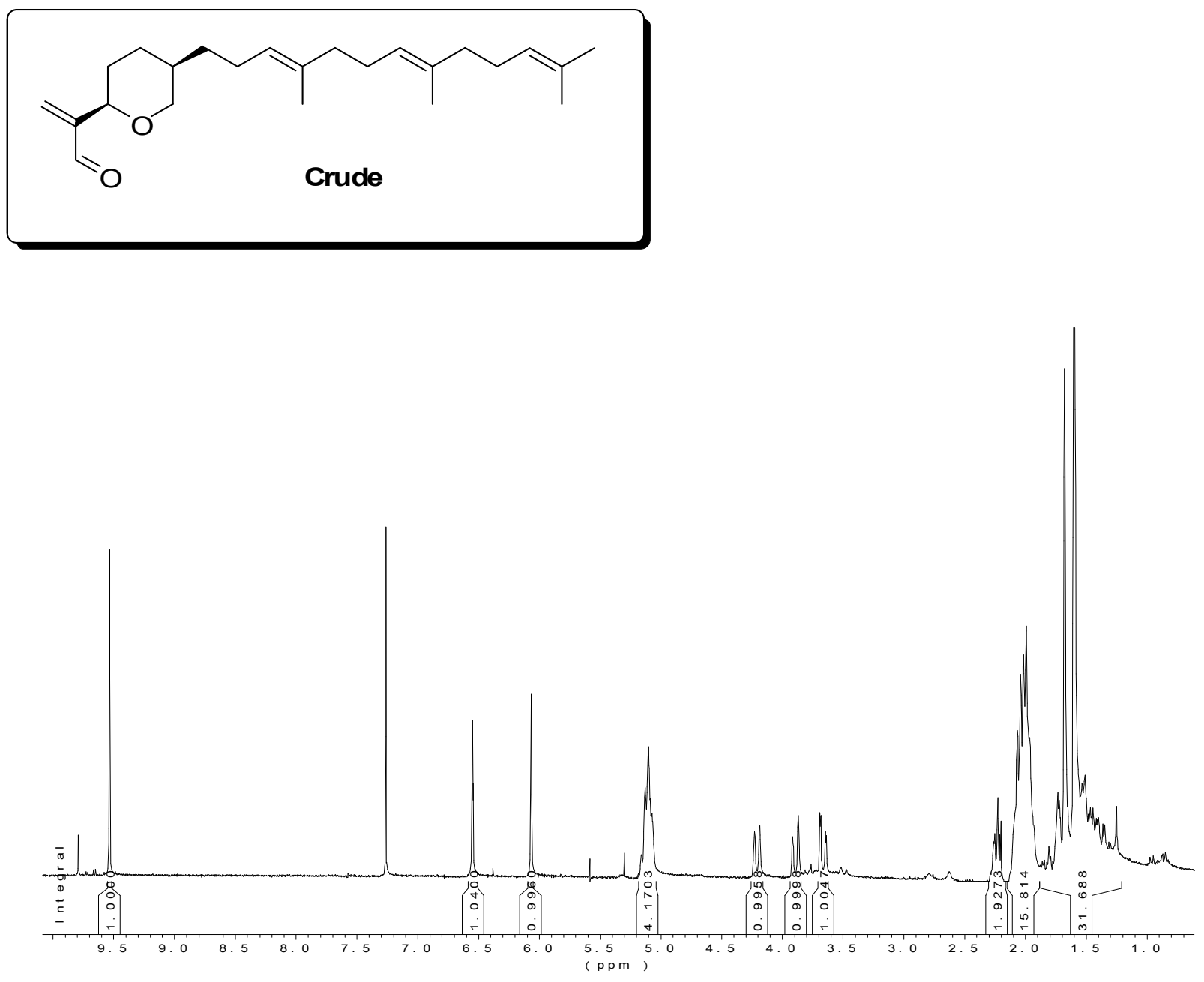

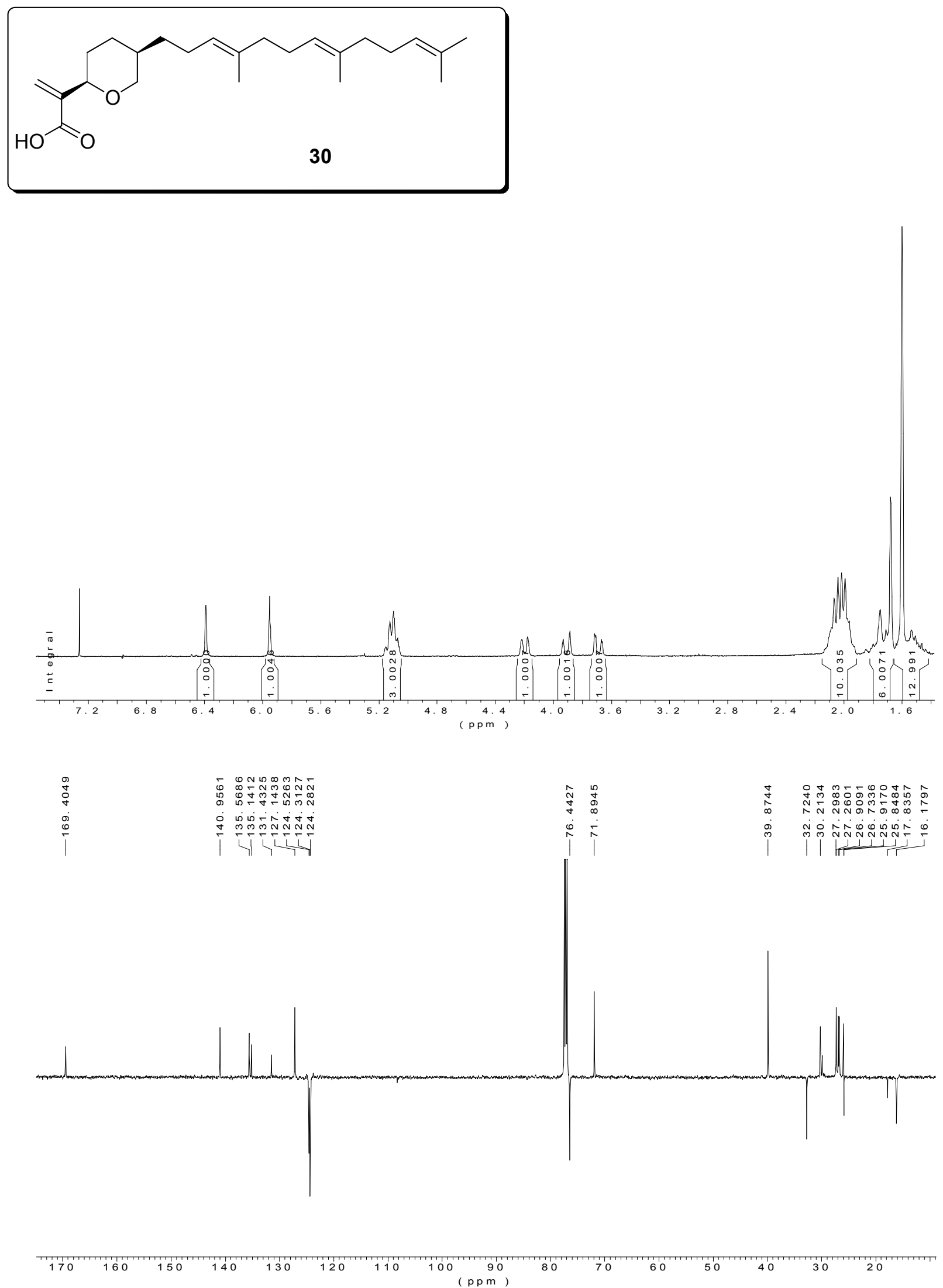


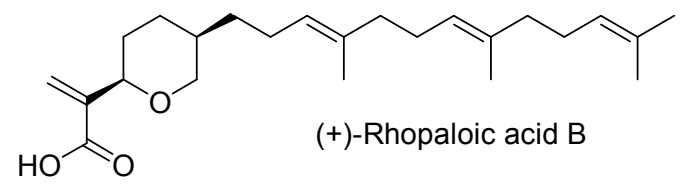

\begin{tabular}{|c|c|c|c|}
\hline${ }^{1} \mathrm{H}$ Literature & ${ }^{1} \mathrm{H}$ & ${ }^{13} \mathrm{C}$ Literature & ${ }^{13} \mathrm{C}$ \\
\hline \multirow{9}{*}{$\begin{array}{c}3.69, \mathrm{dd}, 11.0,2.7 \mathrm{~Hz}, 1 \mathrm{H} \\
2.07, \mathrm{~m}, 4 \mathrm{H}- \\
2.03, \mathrm{~m}, 2 \mathrm{H} \\
1.99, \mathrm{~m}, 4 \mathrm{H}\end{array}$} & $6.39, \mathrm{br} \mathrm{s}, 1 \mathrm{H}$ & 169.9 & 169.4 \\
\hline & $5.95, \mathrm{t}, 1.0 \mathrm{~Hz} 1 \mathrm{H}$ & 141.0 & 141.0 \\
\hline & $5.17-5.04 \mathrm{~m}, 3 \mathrm{H}$ & 135.4 & 135.6 \\
\hline & $4.21, \mathrm{~d}, 10.1 \mathrm{~Hz}, 1 \mathrm{H}$ & 135.0 & 135.1 \\
\hline & $3.90, \mathrm{~d}, 11.5 \mathrm{~Hz}, 1 \mathrm{H}$ & 131.2 & 131.4 \\
\hline & \multirow{2}{*}{$3.69, \mathrm{dd}, 11.5,2.0 \mathrm{~Hz}, 1 \mathrm{H}$} & 127.0 & 127.1 \\
\hline & & $124.4(2 \mathrm{C})$ & 124.5 \\
\hline & \multirow{3}{*}{$2.14-1.91, \mathrm{~m}, 10 \mathrm{H}$} & 124.2 & $124.3(2 C)$ \\
\hline & & 76.1 & 76.4 \\
\hline $1.75, \mathrm{~m}, 2 \mathrm{H}-$ & & 71.7 & 71.9 \\
\hline 1.73, $\mathrm{m}, 1 \mathrm{H}$ & $1.82-1.65, \mathrm{~m} \mathrm{3H}$ & $39.7(2 \mathrm{C})$ & $39.9(2 \mathrm{C})$ \\
\hline $168 \leq 3 \mathrm{H}$ & \multirow{9}{*}{$\begin{array}{c}1.68, \mathrm{~s}, 3 \mathrm{H} \\
1.64-1.40, \mathrm{~m}, 4 \mathrm{H} \\
1.60, \mathrm{~s}, 9 \mathrm{H}\end{array}$} & 32.6 & 32.7 \\
\hline 1.64.m. $1 \mathrm{H}$ & & 30.1 & 30.2 \\
\hline $1.60, \mathrm{~s}, 9 \mathrm{H}$ & & $27.2(2 \mathrm{C})$ & $27.3(2 \mathrm{C})$ \\
\hline $1.55, \mathrm{~m}, 2 \mathrm{H}$ & & 26.8 & 26.9 \\
\hline \multirow[t]{5}{*}{$1.49, \mathrm{~m}, 1 \mathrm{H}$} & & 26.6 & 26.7 \\
\hline & & 25.8 & 25.9 \\
\hline & & 25.7 & 25.8 \\
\hline & & 17.7 & 17.8 \\
\hline & & $16.0(2 \mathrm{C})$ & $16.2(2 \mathrm{C})$ \\
\hline
\end{tabular}

\footnotetext{
: Grais, H. -J., Jurgen, M., Jadhav, V. J. Org. Chem. 2001, 66, 3384 - 3396

ii: M. Muehlbacher, C. D. Poulter, J. Org. Chem. 19911, 53, 1026-1030.

iii: Dixon, D. J. , Ley, S. V., Reynolds, D. J. Chemistry-A European Journal, 2002, 8, 1621-1636.

iv :Lin H.S., Paquette L.A., Synth. Commun., 1994, 24, 2503.

v: Van der Louw, J.; Van der Bann, J.L.; Gerardus, J. J. Out; Franciscus J.J. de Kanter, Friedrich. B.; Gerhard, W. K. Tetrahedron 1992, 45, 9901 and references cited therein.

vi: Wasser, J., Nambu, H., Carreira, E. M. J. Am. Chem. Soc., 2005, 127, 8294-8295.

vii. Achmatowicz, B., Kabat, M. M., Krajewski, J., Wicha, J. Tetrahedron, 1992, 48, 10201-10210

viii. (a) Schaus, S.E., Brandes, B.D., Larrow, J.F., Tokunaga, M., Hansen, K.B., Gould, A.E., Furrow, M.E., Jacobsen, E.N. J. Am. Chem. Soc. 2002, 124, 1307-1315. (b) S.P. Romeril, V. Lee, J.E. Baldwin, T.D.W. Claridge, B. Odell, Tetrahedron Lett. 2003, 44, 7757-7761.

ix: Scwier, J.R., Brown, H.C. J. Org. Chem. 1993, 58, $1546-1552$.

×: Yanai, M., Ohta, S., Ikegami, S., Tetrahedron, 1998, 54, 15607-15612.
} 